OPEN ACCESS

Edited by:

Alejandro Reyes,

University of Los Andes,

Colombia

Reviewed by:

Josue Castro,

University of Copenhagen,

Denmark

Thomas Sutton,

University College Cork, Ireland

*Correspondence:

Eleanor Jameson

Eleanor.Jameson@warwick.ac.uk

Specialty section:

This article was submitted to

Microbiome in

Health and Disease,

a section of the journal

Frontiers in Cellular

and Infection Microbiology

Received: 17 December 2020

Accepted: 19 May 2021

Published: 04 June 2021

Citation:

Townsend EM, Kelly L, Muscatt G, Box JD, Hargraves N, Lilley D and Jameson E (2021) The Human Gut

Phageome: Origins and Roles

in the Human Gut Microbiome.

Front. Cell. Infect. Microbiol. 11:643214.

doi: 10.3389/fcimb.2021.643214

\section{The Human Gut Phageome: Origins and Roles in the Human Gut Microbiome}

\author{
Eleanor M. Townsend ${ }^{1}$, Lucy Kelly ${ }^{1}$, George Muscatt ${ }^{1}$, Joshua D. Box ${ }^{1}$, \\ Nicole Hargraves ${ }^{1}$, Daniel Lilley ${ }^{2}$ and Eleanor Jameson ${ }^{1 *}$ \\ 1 School of Life Sciences, The University of Warwick, Coventry, United Kingdom, ${ }^{2}$ Warwick Medical School, The University of \\ Warwick, Coventry, United Kingdom
}

The investigation of the microbial populations of the human body, known as the microbiome, has led to a revolutionary field of science, and understanding of its impacts on human development and health. The majority of microbiome research to date has focussed on bacteria and other kingdoms of life, such as fungi. Trailing behind these is the interrogation of the gut viruses, specifically the phageome. Bacteriophages, viruses that infect bacterial hosts, are known to dictate the dynamics and diversity of bacterial populations in a number of ecosystems. However, the phageome of the human gut, while of apparent importance, remains an area of many unknowns. In this paper we discuss the role of bacteriophages within the human gut microbiome. We examine the methods used to study bacteriophage populations, how this evolved over time and what we now understand about the phageome. We review the phageome development in infancy, and factors that may influence phage populations in adult life. The role and action of the phageome is then discussed at both a biological-level, and in the broader context of human health and disease.

Keywords: gut microbiome, bacteriophages, phage, metagenomics, isolation, biofilm, disease, diet

\section{INTRODUCTION}

In the past decade, the importance of the human gut microbiome in both health and disease has come to light. It plays a role in the acquisition of nutrients, defence, and in a range of diseases, from gastrointestinal to neurological. Most of these microbiome studies focus solely on bacterial populations, with fungal, archaeal and viral communities often being neglected. However, it is of vital importance that the viruses of microbes, particularly bacteriophage populations, are not overlooked. These bacteriophages (phages), viruses that solely infect bacteria, are the most numerous viral component within the human body (Reyes et al., 2010; Minot et al., 2011), and are predicted to play a significant role in the maintenance of human health, through processes such as horizontal gene transfer (Camarillo-Guerrero et al., 2021).

Phages were first discovered in the early 1900's simultaneously by d'Hérelle and Twort. At the time they were recognised as an alternative to antibiotic therapy (d'Herelle, 1918; d'Herelle, 1919; 
Twort, 1961), but faded into obscurity in Western medicine, before returning as a tool to improve human health in the face of the current antibiotic resistance crisis (Kortright et al., 2019).

While the majority of research has focussed on utilizing phages for therapeutic and biotechnology purposes, the field of analysing natural phage communities in the gut is relatively young and information on phages is often disregarded within microbial metagenome and metatranscriptome libraries. In order to effectively and safely administer phage therapy it is vital to understand natural phage interactions within the human microbiome. Typically, the investigation of the roles phages play in ecosystems has largely focused on marine and terrestrial environments, while the human body remains underexplored. In part, this may be due to issues arising from ethics surrounding samples of human origin and the difficulty of sampling the gut.

Bacteria are the most abundant cellular organisms in the gut. The gut is a nutritionally rich environment which allows bacterial concentrations of close to the theoretical maximum in the order of $10^{7}$ to $10^{14}$ cells, depending on the exact location (Sender et al., 2016). Therefore, there are an abundance of hosts available for phages to infect, which is of importance given the vast majority of viruses within the gut are phages. Together, the total community of phage populations is known as the phageome.

In this review we will discuss what is known about: how phages enter the body, how we study the phageome, its main components, and the important roles and functions it performs in both human health and disease.

\section{METHODS TO IDENTIFY AND CHARACTERIZE PHAGES IN THE GUT}

Since the early work of d'Hérelle (1921), many vital questions within phage biology, and specifically the role in ecosystems such as the human gut, remain unanswered. Techniques used for identifying and characterizing phages in the human gut can be understood from three perspectives: culture-dependent methods, culture-independent methods, and sequencing-based methods. Before the rise of next-generation sequencing in the last 15 years, culture-based methods were the primary approach for researching phage ecology, these rely on isolation techniques to extract virions for investigation (Figure 1) (Mokili et al., 2012).

\section{Culture-Dependent Methods}

For culture-based methods it is necessary to isolate virus-like particles (VLPs) from their complex in situ environment. This is often performed on faecal samples due to the ease and minimal cost in obtaining them. However, the bacterial community of the mucosa-associated microbiome is dominated by different phyla to those found in faeces (Lepage et al., 2005), so much so that it is compositionally distinct (Gillevet et al., 2010). Thus, sampling of faeces may select a combination of shed mucosal bacteria and nonadherent luminal populations (Eckburg et al., 2005). This sampling effect is likely to also affect the phage communities recovered. The relative understanding of phageome composition from faecal sampling is visualised in Figure 2, which highlights gaps in our knowledge from the acquisition of phages through diet, to the various positions of the digestive system. We suggest that future research efforts work towards a more holistic view of the human gut phageome, which undoubtedly relies on colonoscopies for the targeted sampling of the ileum and colon. This will facilitate the study of the phage populations in active luminal and mucosal-associated communities, in order to describe compositional differences in the respective phageomes, and to understand their possible in situ influence on the overall gut microbiome and the human host health.

Three approaches are taken to isolate phages from heterogeneous samples: extraction and purification of virus-like particles (VLPs), enrichment of phages in bacterial host cultures, and induction of temperate phages from lysogenised hosts. Extraction and purification of VLPs is the most common approach, which we describe below.

Following the suspension of faecal matter, intact VLPs can be separated from other microorganisms and free DNA in the faecal suspension by multiple methods, often used in combination (Thurber et al., 2009; Castro-Mejia et al., 2015; Kleiner et al., 2015). Conventional size-fractionation filtration to separate out phages introduces bias against larger VLPs, highlighting the need for novel methods (Serwer et al., 2009; Conceicao-Neto et al., 2015). The efficacy of VLP enrichment can be validated with staining and epifluorescence microscopy (see section CultureIndependent Methods). Gut-dwelling phages can subsequently be isolated using gut-specific bacterial strains to screen for infecting phages within the VLP fraction.

Isolating phages that infect bacteria cultured from the same environment has been readily applied to model hosts including Escherichia coli and Bacteroides fragilis (Gantzer et al., 2002). These can then be used to characterize aspects of phage-host biology, such as phage virulence and persistence. Further, isolation and cultivation is essential to characterize phage replication strategies and understand phage gene function, particularly given that we are limited in assigning functions to $\sim 30 \%$ of phage genes (Silveira et al., 2020). While culture-based methods prove useful in isolating candidates for therapeutic and industrial applications (Bonilla et al., 2010; Mattila et al., 2015), the isolation of gut phages for ecological purposes is limited by host selection bias. In fact, it is challenging to culture gut bacterial strains or obtain those already cultured, with many sampling efforts focused on aerobic bacteria associated with disease from a small percentage of the human population.

To overcome this issue, recent progression in the field of culturomics (Lagier et al., 2012), which combines diversified culturing conditions (Lagier et al., 2015) with mass spectrometry (MALDI-TOF) and 16S rRNA sequencing, has helped to identify much of this bacterial dark matter of the gut, once thought to be uncultivable (Lagier et al., 2015). This technique has been used to add many gut bacterial isolates to the repertoire for isolating gut phages (Lagier et al., 2016). For example, the combination of culturing, enrichment and sequencing techniques has led to the discovery and isolation of the widespread human CrAssphage 


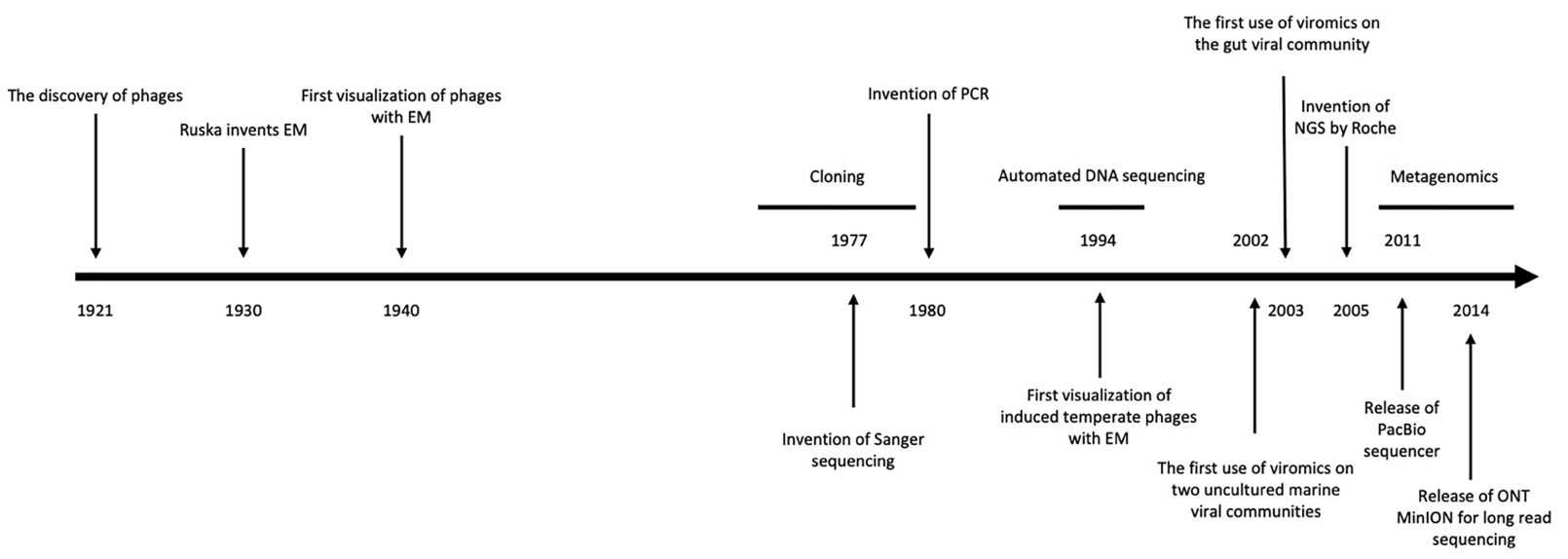

FIGURE 1 Timeline of methodological discoveries. A summarised timeline including the invention of key tools for the study of phages, from their discovery in 1921 to recent modern metagenomic sequencing technologies. Adapted from Mokili et al. (2012).

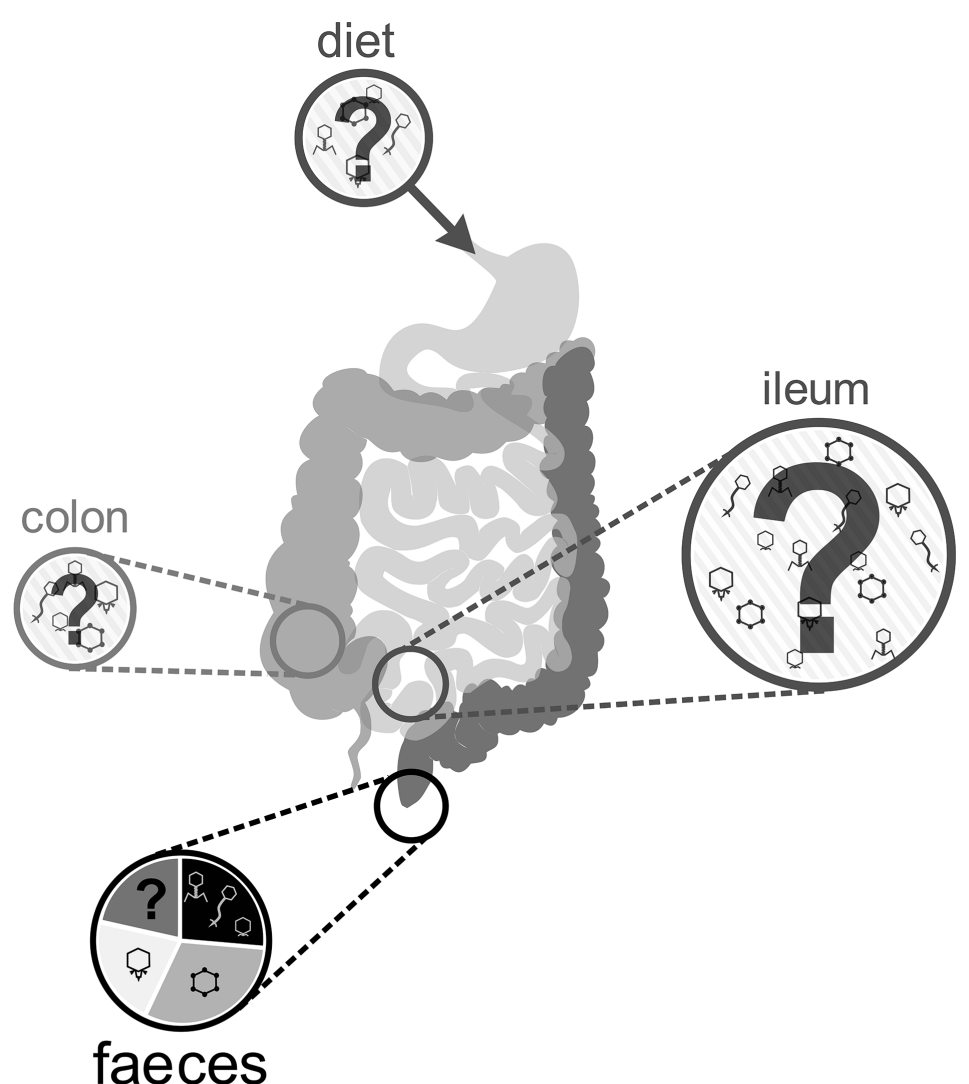

Caudovirales Microviridae $\&$ crAssphage ? Unclassified

FIGURE 2 | Schematic of the human gut, indicating unknown inputs to the gut phageome from diet and the environment, highlighting the largely unknown phage composition of the ileum and colon and indicating the relative proportions of phages in the best understood samples: faeces. Circle size provides a rough guide to the relative abundance of phage particles observed in different gut compartments (Zoetendal et al., 2012; Hoyles et al., 2014; Shkoporov and Hill, 2019), but current understanding is still lacking. Most research activity has focused on faecal samples, represented in the lowest pie chart, depicting the relative proportions of different phage groups identified through metagenome analysis, based on Shkoporov and Hill (2019). 
(see section Identification of Novel Human Phages) (Dutilh et al., 2014; Shkoporov et al., 2018a).

Temperate phages, integrated within their bacterial host genomes as prophages, also pose a challenge for isolation, but can be induced from lysogenised isolates using UV light (Furuse et al., 1983), mitomycin C, and heat treatment (Pelzek et al., 2013). Induced prophages can then be separated from their hosts using the same methods as for virulent phages. Non-lysogenised reporter host strains can then be used to verify temperate phage infectivity. There is debate over the relative importance of virulent and temperate phages in the gut (see section PhageHost Population Dynamics), therefore the isolation of a range of phages with different replication strategies is key to testing these hypotheses.

In addition to characterizing aspects of phage-host interactions, concentrated phage populations can be further interrogated with flow cytometry (Brown et al., 2015), microscopy and molecular biology techniques (Castro-Mejia et al., 2015) (see section Culture-Independent Methods), or sequenced for further in silico analyses (see section Metagenomics). Culture-dependent methods of phage isolation and characterisation focus on individual phage functions and replication strategies. Despite this being vital for the investigation of phage populations and phage-host dynamics, cultureindependent methods have facilitated the study of phageome diversity and function.

\section{Culture-Independent Methods}

While the recent rise in culture-independent methods since the 1980s was due to the advent of Sanger sequencing, these methods arose decades earlier thanks to electron microscopy (EM)
(Figure 1). Phages were first visualised with EM in the 1930s (Peankuch and Kausche, 1940; Ruska, 1940), which circumvented the magnification limitations of light microscopes, allowing for the visualisation of phages. Transmission EM (TEM) was used to observe that tailed phages were the most abundant VLPs in human faeces (Flewett et al., 1974), with recent microscopy indicating that the gut virome is almost exclusively represented by the order Caudovirales (Hoyles et al., 2014; Guerin et al., 2018). A large diversity of viral morphotypes have been visualised within individuals (Hoyles et al., 2014), with most VLPs representing phages. Microscopic techniques have also been used to enumerate gut phages (Lepage et al., 2008; Hoyles et al., 2014), however it has been suggested that TEM and staining could overestimate the true active phageome by up to 8 times (Kleiner et al., 2015). An evaluation of the staining and visualisation techniques that have led to a number of important discoveries is given in Table 1. The revelation of the high abundance of viruses, especially phages, in the human gut as observed by microscopy has paved the way for modern phage research.

Modern molecular biology techniques reflect the biochemical, technological and high throughput advancements made over the past few decades. The use of which have resulted in breakthroughs such as the identification of toxin-antitoxin systems in Lactobacillus johnsonii prophages using recombinant protein gene expression (Denou et al., 2008). The use of molecular biology for gut phage research is evaluated in Table 2.

It is finally worth noting the use of whole animal models for investigating the factors that drive microbiome structure and function, and disease-state modelling of the human gut,

TABLE 1 | Microscopy techniques used to study the gut phageome, including the major advantages and limitations of each technique.

\begin{tabular}{lll}
\hline Technique & Example gut discoveries & Advantages \\
\hline TEM & $\begin{array}{l}\text { Tailed phages were the most abundant VLPS in human } \\
\text { faeces (Flewett et al., 1974). }\end{array}$ & Visualisation of phage morphology. \\
& & \\
& Faecal samples from patients were found to share no & \\
& VLPS (Hoyles et al., 2014). \\
EFM & Up to $5.58 \times 10^{9}$ VLPs were observed per gram of faeces & Enumeration of VLPS in samples. \\
& (Hoyles et al., 2014).
\end{tabular}

Can validate viral purification procedures.

Greater accuracy and speed compared to TEM

\footnotetext{
phageFISH The viability of VLPS can be determined through single cell dynamic measurements, as shown with marine phages (Allers et al., 2013)

CLSM The complex microenvironment and spatiotemporal succession can be studied in multispecies biofilms, as shown with non-phage viruses (Røder et al., 2016).

CryoEM Phage capsids of Bortadella phages were visualised at angstrom resolution, discovering unique protein folds, as shown with non-gut phages (Zhang et al., 2013).
}

PhageFISH is the only non-genetic method to implicate lytic, lysogenic, and chronic phage infection modes (Dang and Sullivan, 2014). Non-destructive sampling. Can be used to visualise the biofilm infection over time.

Very high resolution.

\section{Limitations}

Biased towards identifying tailed phages due to potential loss of tail structures in sample preparation (Williamson et al., 2008). Limited to observations of morphologies. Time-consuming.

VLP counts are conservative estimates of true viral abundances, given the imprecision of visualising single fluorescent dots. Loss of VLPs during preparation and filtration of samples, e.g. large VLPS of the order Megavirales (Colson et al., 2010).

Viability of the VLP to infect and lyse bacterial cells is unknown. VLPs may be membrane vesicles, gene transfer agents or cell debris containing nucleic acids (Forterre et al., 2013).

Limited to biofilms of bacterial species which can be fluorescently labelled.

Destructive sampling. 
TABLE 2 | Summary of molecular biology techniques used to study the gut phageome, including the major advantages and limitations of each technique.

\begin{tabular}{|c|c|c|c|}
\hline Technique & Example gut discoveries & Advantages & Limitations \\
\hline Electrophoresis & $\begin{array}{l}\text { SDS-polyacrylamide gel electrophoresis on a CsCl } \\
\text { fraction of human faeces isolated CrAss-like } \\
\text { phages for subsequent mass spectrometry (Guerin } \\
\text { et al., 2018). }\end{array}$ & $\begin{array}{l}\text { Multiple viral populations can be } \\
\text { separated from multispecies samples } \\
\text { due to their differing capsid sizes. }\end{array}$ & $\begin{array}{l}\text { No taxonomic information can be determined by } \\
\text { electrophoresis alone. }\end{array}$ \\
\hline $\begin{array}{l}\text { Recombinant } \\
\text { protein gene } \\
\text { expression }\end{array}$ & $\begin{array}{l}\text { Toxin-antitoxin systems were identified within } \\
\text { prophages of Lactobacillus johnsonii (Denou et al., } \\
\text { 2008). }\end{array}$ & $\begin{array}{l}\text { Proteins can be overexpressed to } \\
\text { obtain high titres for subsequent } \\
\text { analysis. }\end{array}$ & $\begin{array}{l}\text { Cloning of viral genes into heterologous host systems can } \\
\text { be difficult given that viral genomes often encode modified } \\
\text { nucleotides (Warren, 1980) and lethal genes (Wang et al., } \\
\text { 2010). } \\
\text { Expressed proteins may not be functional due to } \\
\text { misfolding and incorrect modifications in expression host. }\end{array}$ \\
\hline Microarrays & $\begin{array}{l}\text { The new-born infant gut viral community was } \\
\text { found to be dynamic (Breitbart et al., 2008). } \\
50 \% \text { of the strain-specific DNA in Lactobacillus } \\
\text { johnsonii was found to derive from prophages } \\
\text { (Ventura et al., 2003). }\end{array}$ & High-throughput. & $\begin{array}{l}\text { Incompatible with novel viral genomes as a priori } \\
\text { sequence information is required to design probes. } \\
\text { Viral DNA amplification steps prior to microarray analysis } \\
\text { can introduce bias, making relative abundances no longer } \\
\text { reflect that of the sample studied. }\end{array}$ \\
\hline $\begin{array}{l}\text { Single-cell } \\
\text { DNA }\end{array}$ & $\begin{array}{l}\text { Sequencing of commensal gut bacteria can } \\
\text { facilitate the identification of integrated prophages }\end{array}$ & Facilitates taxonomic investigation. & $\begin{array}{l}\text { Requires isolation and cultivation of lytic phages and hosts } \\
\text { of temperate phages. }\end{array}$ \\
\hline sequencing & (Ventura et al., 2003; Denou et al., 2008). & $\begin{array}{l}\text { Can assemble viral genomes for } \\
\text { viruses excluded from metagenomic } \\
\text { approaches (Martinez-Hernandez } \\
\text { et al., 2017). }\end{array}$ & No community-wide view. \\
\hline \multirow[t]{2}{*}{ qPCR } & $\begin{array}{l}77 \% \text { of faecal samples contained phages carrying } \\
\text { at least one antibiotic resistance gene (Quiros } \\
\text { et al., 2014). }\end{array}$ & $\begin{array}{l}\text { Detection and quantification of } \\
\text { specific genes in real time. }\end{array}$ & Sequence of target gene is required a priori. \\
\hline & $\begin{array}{l}\text { Longitudinal tracking of phage and bacterial hosts } \\
\text { in the faeces of a mouse model system facilitated } \\
\text { the study of predator-prey dynamics (Hsu et al., } \\
\text { 2019). }\end{array}$ & & $\begin{array}{l}\text { Nonspecific binding of template can lead to amplification } \\
\text { of off-target genes. }\end{array}$ \\
\hline \multirow[t]{2}{*}{$\begin{array}{l}\text { Viral tagging \& } \\
\text { flow cytometry }\end{array}$} & $\begin{array}{l}363 \text { unique phage-host pairings were predicted, } \\
\text { including many uncharacterised phages (Džunková } \\
\text { et al., 2019). }\end{array}$ & Infer phage-host relationships. & $\begin{array}{l}\text { Direct evidence of successful phage infection is not } \\
\text { provided by attachment of phage and host cell. }\end{array}$ \\
\hline & & $\begin{array}{l}\text { Culture-independent. } \\
\text { Flow cytometry has the facility to sort } \\
\text { individual phage-host pairs for } \\
\text { downstream sequencing etc. }\end{array}$ & $\begin{array}{l}\text { Different assay conditions can bias the phage-host } \\
\text { pairings observed from the community. }\end{array}$ \\
\hline
\end{tabular}

e.g. inflammatory bowel diseases (see section Role of Bacteriophages in Human Disease). Animal models enable the experimental investigation of phage-host interactions, to develop hypotheses that connect alterations in the gut microbiome to disease. An extensive review of simple animal models of the gut microbiome was written by Douglas (2019). Murine models have been applied to a wide range of phage studies, including phagehost population dynamics over time (Hsu et al., 2019), changes in phage populations after antibiotic exposure (Modi et al., 2013), investigating the role and function of phage proteins (Miernikiewicz et al., 2016), and the abundance and activity of prophages in the gut (Kim and Bae, 2018). However, there has been much critique of the use of mice models to represent the human gut, discussed in Nguyen et al. (2015). Animal models have enabled hypothesis generation and testing, but due to differences in their physiology and natural microbiota, they should be used as a stepping-stone rather than as final evidence to inform human microbiome related medicine.

\section{Metagenomics}

Metagenomics is the most recent development on the gut phageome methods timeline, facilitated by the advent of highthroughput sequencing (Figure 1). Metagenomics provides a snapshot of the DNA phage community: the populations that can be recovered, and the genes they encode. There are two favoured approaches to performing metagenomics: sequencing of the entire nucleic acid complement through shotgun metagenomics, and amplification of marker genes such as the $16 \mathrm{~S}$ ribosomal RNA gene in amplicon sequencing (Jovel et al., 2016; Hayes et al., 2017). Given the absence of a universal viral marker gene, amplicon approaches are unviable for studying the phageome, although some group-specific markers do exist for estimations of phage diversity (Adriaenssens and Cowan, 2014). Instead, whole-community metagenome (WCM) sequencing has been demonstrably the most successful method for exploring phage communities across environmental niches (Hayes et al., 2017). Metagenomic sequencing of gut microbiome free-phages relies on enrichment of VLPs, prior to nucleic acid extraction, introducing the previously discussed biases. Protocols typically use size fractionation of free phages selecting for $<200 \mathrm{~nm}$, with particular focus on DNA phages (Kleiner et al., 2015). A review of the steps involved in producing phage metagenomes, including the biases associated with each step, has been reviewed by Hayes et al. (2017).

The first use of viral metagenomics (viromics) on the human gut described phages found in human faeces, finding evidence for 
the dominance of DNA phages (Breitbart et al., 2003). Since then, many studies have aimed to identify phages from gut WCMs (Ogilvie et al., 2013; Waller et al., 2014). While metagenomes are expected to be dominated by bacterial DNA, it has been shown that up to $17 \%$ of gut microbial DNA is predicted to be of phage origin (Ogilvie et al., 2013). The phage sequences recovered are likely to be predominantly prophages, given the abundance of bacterial DNA in gut metagenomes (Williamson et al., 2008; Stern et al., 2012). This is not a limitation, as the gut phageome of humans and mice is dominated by integrated prophages, carried by the majority of intestinal bacteria (Waller et al., 2014; Kim and Bae, 2018). Nonetheless, analysing both the viral metagenome (virome) and WCMs from faecal samples is essential to appreciate the total phage community occupying the gut niche. Furthermore, it is particularly important for deciphering the presence of a core gut phageome.

Phage populations recovered through viromics do not necessarily reflect the true diversity of the gut phageome; larger phage genomes are often only assembled for the most abundant viral genotypes present within a sample, and many lowly abundant viral species are likely to be lost in purification steps during sample preparation or biased against by the sequencing protocol. In addition, the phylogenetic binning strategy for reconstructing short reads in shotgun metagenomes may result in the loss of closely related phage contigs due to high nucleotide sequence similarity. Genome assembly and binning is complicated by mosaicism within phage genomes, a common feature of prophages (Lima-Mendez et al., 2011), which are of particular interest in the gut phageome (Waller et al., 2014; Kim and Bae, 2018). Given this, and the costs associated with achieving high sequencing depths from faecal samples, many contigs represent only fragments of the true sequenced gut phage genomes (Shkoporov and Hill, 2019). To mitigate issues with the accuracy of viral population detection, the research community has defined thresholds of contig length and read coverage to balance sensitivity with false-discovery rate (Roux et al., 2017). This includes considering phage populations as "detected" only when virome reads map at $\geq 90 \%$ nucleotide identity to nonredundant contigs $\geq 10 \mathrm{~kb}$ over $\geq 75 \%$ of their length. Subsequent false-discovery rates have been shown to remain low at $0.2 \%$ using these thresholds (Roux et al., 2017). Further, software tools such as CheckV (Nayfach et al., 2020) can be integrated into viromics pipelines to estimate the confidence in assembled phage genomes for downstream analyses (Table 3). A confidence score of $>90 \%$ completeness indicates high quality contigs, important for distinguishing short contigs as nearcomplete phage genomes. CheckV can also be used to identify integrated prophages through the detection of flanking host genes (Nayfach et al., 2020).

As a consequence of the above limitations, and the exclusion of single-stranded DNA (ssDNA) and RNA phages whose true

TABLE 3 | Bioinformatic tools used to study the gut phageome, including techniques, research questions they may address and name of the software.

\begin{tabular}{lll}
\hline Technique $\quad$ Research questions & Tools
\end{tabular}

cation

Annotate Do the phage genomes encode Prodigal (Hyatt et al., 2010) or PHANOTATE (McNair et al., 2019) to predict coding sequences.

genomes auxiliary metabolic genes (AMGs)?

Do the phage genomes encode Prokka (Seemann, 2014) or eggNOG (Powell et al., 2012), to functionally annotate draft viral taxon-specific genes? Are the phages temperate or virulent? genomes.

Hmmscan (Eddy, 2011), to align protein sequences to databases (e.g. pVOGs (Grazziotin et al., (Clooney et al., 2019) 2016) and Pfam/vFam (Skewes-Cox et al., 2014; El-Gebali et al., 2019) and Genecatalog (Li et al., 2014).

Is there a global phage gene Phyre2 (Kelley et al., 2015), to find proteins with structural homology . pool?

Phage Are the genomes assembled DRAM-v to predict putative AMGs associated with metabolism (Shaffer et al., 2020).

prediction phage genomes? De-novo prediction of phages using tools such as VirFinder/ DeepVirFinder (Ren et al. 2017; Ren et al., 2002) and VirSorter2 (Guo et al., 2021) Confirmation of viral genome completeness with CheckV (Nayfach et al., 2020) and IMGNR v3 (Roux et al., 2021).

How diverse are the phages? Alignment to hallmark taxon-specific marker genes. Are any known viral taxa predicted?

BLAST (Camacho et al., 2009), PhymmBL (Brady and Salzberg, 2009) and k-mer approaches (Trifonov and Rabadan, 2010), for taxonomic assignment.

Comparison to gut viral databases, e.g. Gut Virome Database (GVD) (Gregory et al., 2020).

(Waller et al., 2014) (VazquezCastellanos et al., 2014)

How similar are they to phages in other ecosystems? Standley, 2013). De-novo classification with vConTACT2 (Zablocki et al., 2019).

Phage-host Which gut hosts do the phages Genetic homology of integration sites and CRISPR spacer sequences between phage and host. relationships infect?

Is phage predation species or Phage genome signature-based recovery.

(Stern et al., 2012) strain specific?
(Ogilvie et al., 2013)

Identification of integrated prophage.

Abundance profiles.

De-novo relationships with WIsH (Galiez et al., 2017)

(Dutilh et al., 2014) 
prevalence in the gut phageome is unknown, analysis of phage diversity through metagenomics often underestimates absolute viral richness. Further, inferences on phage diversity yield only a snapshot of the sampled phage community at a given time point. The temporal dynamics of the gut microbiome can only be understood by sampling multiple time points, which adds logistical challenges of recalling participants, costs, and is both more lab- and bioinformatically-intensive due to the size of the datasets assembled and analysed. Nonetheless, there are a few examples of longitudinal studies of the gut phageome, which are discussed in section Development of the Phageome (Minot et al., 2013; Shkoporov and Hill, 2019).

There are a number of tools used to describe and interrogate environmental phageomes through metagenomics, reviewed in Table 3. Many of these tools are alignment-based, requiring sequence homology between genes and genomes for annotation, phage classification and the inference of phage-host relationships. Historically, due to the underrepresentation of viruses in reference databases, in conjunction with a high proportion of novel phages sequenced from the gut (Shkoporov et al., 2018b), alignment-based approaches were limited. It has been reported that $>75 \%$ of reads from gut viromes did not align with known viral genomes (Aggarwala et al., 2017). In another study, $<2 \%$ of the viral genomes assembled from human faeces could be assigned to known taxa (Shkoporov et al., 2018b). Additionally, both RNA (Callanan et al., 2020) and non-tailed (Kauffman et al., 2018) phages are significantly underrepresented in phage databases. Therefore, alternative tools that do not rely on similarity to databases have been proposed to circumvent these limitations (Reyes et al., 2012), but have yet to see widespread implementation. Recently, great advances have been made in assembling comprehensive databases for the detection of viral populations from gut microbiome studies, e.g. Gut Virome Database (Gregory et al., 2020). A combination of this increased viral representation in databases, software advances, and the development of novel bioinformatic tools (Table 3), is essential for continuing to improve the proportion of true phageomes captured by viromic analysis and understanding their functions.

Another limitation associated with assembled viral genomes is the lack of information on viral activity (Stern et al., 2012). Sequenced phages may be quiescent and thus less metabolically important relative to the active phage community. Further, the verification of host range, gene function, and replication strategy characterisation requires the cultivation of phage isolates. The activity of phages can, however, be resolved through a combination of 'omic strategies. In a similar approach to sequencing the DNA metagenome, other meta-'omic strategies have been adopted to study the gut phageome at the RNA, protein and metabolite-levels.

\section{Other 'Omics}

In metatranscriptomics, the sequencing and de-novo assembly of RNA contigs is carried out in a similar way to metagenomics, reviewed for environmental samples in (Lim et al., 2013). Notably, for recovering RNA phages from the gut, there are inherent difficulties facing the collection, storage and assembly of
RNA libraries from human samples, which have been reviewed by Bikel et al. (2015). This could be why metatranscriptomics has had limited usage in the study of the gut phageome. In addition, tools developed for the prediction of DNA phage contigs from metagenomes are not optimised for the prediction of RNA phages, due to the scarcity of known RNA phages and the vast diversity in their genomes (Duffy et al., 2008). Subsequently, RNA phages have rarely been identified in the gut; the RNA viruses that have been identified were derived from plants consumed in the diet (Zhang et al., 2005; Lim et al., 2015). A novel alternative approach to recovering RNA phages, based on homology to known core single-stranded RNA (ssRNA) phage genes, has been implemented on metatranscriptomes generated from activated sludge and aquatic environments (Callanan et al., 2020). Future studies should aim to use this method to recover RNA phages from the gut, given recent findings of their abundance and activity in other ecosystems (Callanan et al., 2018; Starr et al., 2019).

A second use of metatranscriptomics comes with its combined implementation alongside metagenomics. By mapping metatranscriptome reads to assembled phage genomes, gene transcription can be estimated as a proxy for phage activity to discern active from dormant prophages. Further, the most actively transcribed genes can be determined for the functional analysis of the phageome. When this combined approach is applied to samples over a time-course, the dynamics and persistence of phage infection can be determined, as shown for marine ecosystems (Sieradzki et al., 2019). To the best of our knowledge, metatranscriptomics has had limited implementation on the gut phageome, but future efforts should enable the investigation of active functional genes, including auxiliary metabolic genes, and discern the proportion of the phageome that is active within the gut microbiome in diseased and healthy states. This could uncover further evidence for implicating the phageome in disease.

Metaproteomics captures the protein complement of microbial communities and is a direct study of the end product of gene expression, used to evaluate the functional state of a community. Downstream analytical techniques such as mass spectrometry and nuclear magnetic resonance can be implemented to characterize isolated proteins. Modern advances in these techniques have improved the throughput and accuracy of this approach for its application on the gut microbiome (Arnold et al., 2016). Metaproteomics has been used to provide further support for the dominance of temperate phages in the gut, from an abundance of virally-encoded proteins associated with lysogenic replication strategies (Ogilvie et al., 2013). Additionally, metaproteomics has revealed phage proteins that were not identified through genome-based alignment approaches, indicating the advantage of a complementary multi-'omic strategy (Ogilvie et al., 2013). Other applications of proteomics on the gut phageome are very rare in the literature, but have been proposed to uncover the functional potential of phages (Shkoporov et al., 2019). Like metatranscriptomics, future metaproteomic analysis should enable inferences on the relative functional state of the phageome in the gut through comparative and longitudinal studies. 
Metabolomics specifically targets the metabolites present within cells, thus including those involved in lysogenic conversion. In gut microbiome research, metabolomics has been used to evaluate the metabolic state of the gut microbiome as a result of phage predation (Hsu et al., 2019). This approach facilitates the direct monitoring of phage function in the gut, moving beyond the correlation of phage populations with those of their host. Future use of metabolomics could be applied to explore the role phages play in microbial community function, interactions with the human host and differences in phage-mediated community function between healthy and diseased states (see section Role of Bacteriophages in Human Disease).

The methods used to identify and characterize phages have come a long way since phages were first visualised in 1940 (Figure 1). Early culture-dependent methods isolated intestinal phages from faeces for the first time, microscopy enabled the morphotypes and abundances of gut phages to be realised, and recent 'omic advances have facilitated the study of phageome diversity and function.

There are undoubtedly numerous additional challenges that face the study of the phageome, as compared to the bacterial microbiome. While we have provided many limitations of the existing methods, we have underlined the potential for their future development and implementation in studying the gut phageome. Namely, the greatest challenge involves expanding the proportion of the phageome captured by 'omic analyses. This means recovering increasingly low abundant phage populations and describing their functional potential and reality. To this aim, future advances in both culture-dependent and sequencing methods, in addition to the combination of these approaches, can be achieved in a number of ways. This begins with amending sampling biases through the direct study of gut samples, and the greater representation of sampled individuals outside of Europe and North America. Increasing sequencing depth, augmenting bioinformatic tools for recovering and annotating phage contigs, and combining 'omic techniques will maximize our ability to characterize sampled phageomes. The simultaneous implementation of culture-dependent methods with sequencing approaches can validate in silico hypotheses of phage-host interactions for implicating phage populations in the overall functioning of the gut.

\section{COMPOSITION OF THE GUT PHAGEOME}

\section{Development of the Phageome}

The human gut is host to a complex community of microorganisms that contribute greatly to gut function, immune responses and disease pathogenesis. The human microbiome develops from birth, is predominantly of maternal origin, and expands rapidly in response to many environmental factors (Yassour et al., 2016). The phageome follows a similar pattern to the bacterial populations, with changes in diversity occurring within the first 2 weeks of life (Figure 3) (Breitbart et al., 2008). Initially the phageome is of low diversity, with the sources of inoculation being unclear (Mills et al., 2013). Suggestions of the origin of this initial phageome include dietary, environmental and maternal sources (Breitbart et al., 2008), and induction of prophages from the newly colonised
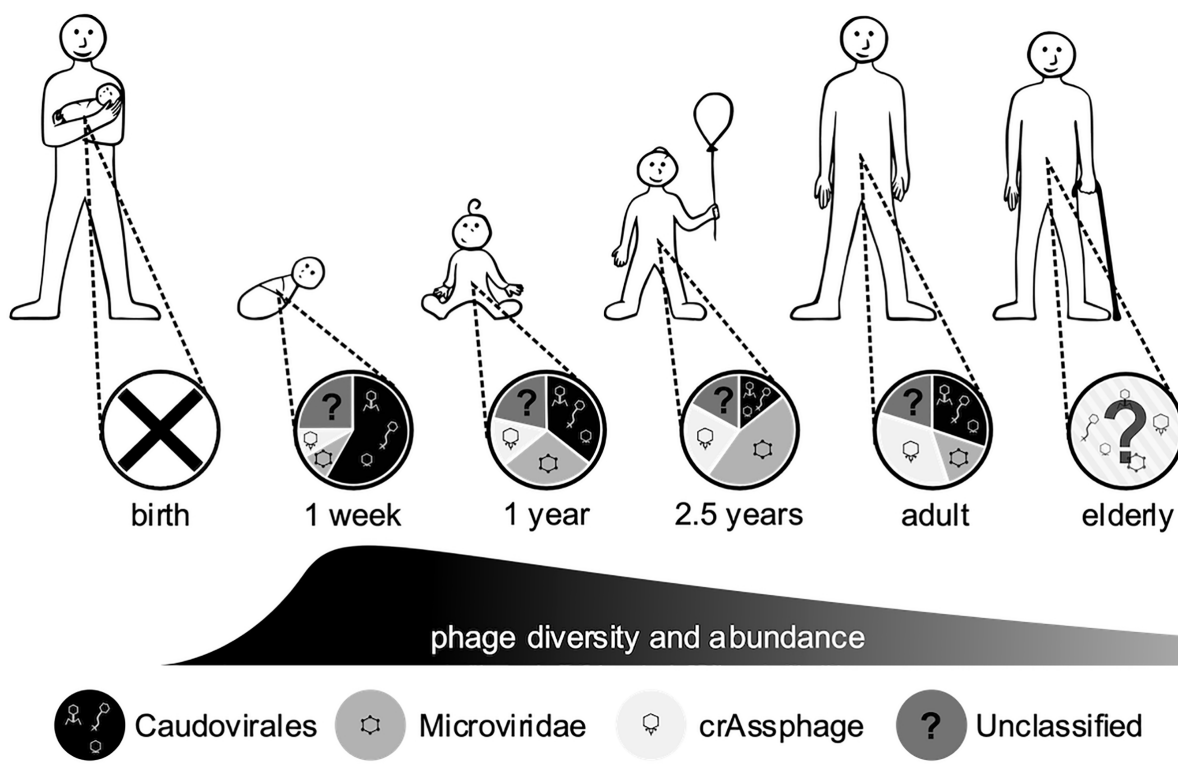

FIGURE 3 | Changes in the gut phageome over the human lifetime. Pie charts represent the observed ratios of different phage groups at discrete sampling times, from birth where no endemic phages were observed to adults, while the humped line describes changes in phage diversity and abundance over time, which both peak in the weeks after birth (Breitbart et al., 2008; Reyes et al., 2010; Kim et al., 2011; Lim et al., 2015; McCann et al., 2018). 
microbiota (Breitbart et al., 2003). As early bacterial colonisers are also derived from the maternal source, these should not be considered distinct sources but rather a multifaceted acquisition. It has been reported that vaginal delivery or caesarean section can produce distinctly different viral communities within the infant gut phageome (McCann et al., 2018). Bifidobacterium and their phages can be vertically inherited from the mother via breastmilk (Duranti et al., 2017), including prophage induced from colonizing bacteria (Liang et al., 2020) and phage that infect probiotic lactic acid bacteria abundant in breastmilk (Martin et al., 2003; Pannaraj et al., 2018). Sharon et al. (2013) found that a group of closely related Caudovirales bloomed, then persisted in the infant gut along with their host strains, between weeks 15-24 after birth. These Caudovirales initially dominate the gut phageome, then a shift to a Microviridae-dominated population occurs at around two and a half years old (Figure 3) (Lim et al., 2015). It has been postulated that the Caudovirales populations cannot be supported by the minimal bacterial community, leading to a phage population crash. This, in turn, allows bacterial populations to thrive without predation, shifting the microbiome towards a mature state with an increased diversity of bacteria.

In adulthood, the gut phageome has been proposed to be dominated by phages exhibiting a temperate lifestyle (Silveira and Rohwer, 2016). While this may be true, measures of the phageome are not absolute and the diversity of temperate phages may be masked by a few highly abundant lytic phages, meaning that the intricacies of the phageome have yet to be fully resolved (Sutton and Hill, 2019). The adult gut phageome shows a return to a Caudovirales-dominant population with a subpopulation of Microviridae (Figure 3) (Kim et al., 2011; Manrique et al., 2016), although there is a large degree of inter-individual variation (Reyes et al., 2010). Communities dominated by Microviridae or CrAssphage have also been described in some individuals (Shkoporov et al., 2019). This inter-individual diversity appears to be driven by environmental factors (Reyes et al., 2010), and is significant even between identical twin infants and families (Lim et al., 2015; Reyes et al., 2015). Some RNA phages have also been detected in the gut phageome, but in significantly lower abundance in comparison to DNA phages (Breitbart et al., 2003; Zhang et al., 2005). However, it is difficult to distinguish if this is due to a genuine reduced presence or lack of effective techniques to detect RNA phages, as discussed in section Other 'Omics. Later in life, the gut microbiome decreases in diversity and shifts from a Firmicutes- and Bacteroidetes-dominated community to one dominated by obligate and facultative anaerobes (Nagpal et al., 2018). While it has yet to be investigated, the functional capacity of the phageome may also be influenced by this change in composition. Though the gut phageome seems to be highly specific from person-to-person, there has been indication of stability over time (Shkoporov et al., 2019), with sequences of the same phages detected more than 2 years apart (Minot et al., 2011; Minot et al., 2013). There is emerging evidence for a core gut phageome, Manrique et al. (2016) identified a set of 23 phages that were present in over $50 \%$ of healthy gut samples analysed, with an additional 44 phage groups present in $20-50 \%$ of individuals, suggesting a secondary set of shared phages. A recent study found that both CrAssphage and Microviridae were the two most stable members of the gut viral community, suggesting a potential role as part of a core phageome (Shkoporov et al., 2019). However, any core communities only represent a fraction of the overall population, it is almost impossible to simplify the complexity and individuality of the phageome.

\section{Identification of Novel Human Phages}

CrAssphage were discovered in 2014 via the cross-assembly (crAss) method, providing the name CrAssphage. Previously undetected, they are the most abundant gut phage currently known (Dutilh et al., 2014). Through both metagenomic and culture-based studies, their host has been predicted to be Bacteroides, with a podoviridae-like morphology (Shkoporov et al., 2018a; Yutin et al., 2018). Interestingly, no known lysogeny genes were found in the genome of the original CrAssphage isolate (crAss001), yet the phage could maintain its population in culture with Bacteroides intestinalis, in part due to the long latent phase and very small burst size of 2.5 plaque forming units per cell. However, the true mechanism was not elucidated.

There is evidence to suggest that CrAssphage has long been a member of human gut phage communities, having also been found in the gut of non-human primates, such as baboons and gorillas (Edwards et al., 2019). This presents the idea that CrAssphage have evolved with humans and maintained their presence in the gut phageome. Furthermore, CrAssphage sequences have been found to persist universally across the globe. The majority of strains identified were only present in individual samples, however one strain was identified in 104 independent samples spread across multiple continents (Edwards et al., 2019). In addition, CrAssphage was more abundant in the gut of industrialised populations in comparison to traditional hunter-gatherer populations (Honap et al., 2020). These findings, taken together, suggest a recent human expansion event, associated with industrialisation, led to the expansion of CrAssphage within the human population.

The presence of CrAssphage in the gut appears to begin in infancy (McCann et al., 2018; Edwards et al., 2019; Shkoporov et al., 2019). It appears to be transmitted vertically, with homology found between isolates from mother and child (Siranosian et al., 2020), which also corresponds with the presence of its Bacteroides host (Tanaka and Nakayama, 2017). However, there is again a large degree of inter-individual variation in the presence and abundance of CrAssphage.

A group of megaphages (with genomes $>500 \mathrm{~kb}$ in length) have been identified from human faecal samples and have been tentatively predicted to infect Prevotella (Devoto et al., 2019). These "Lak phages" were detected in a wide range of samples and are exceptional, not only for the size of their genomes but also, for their use of a genetic code with an alternative stop codon, setting them apart from other phage families (Devoto et al., 2019). Lak phages are yet to be isolated, but the size of their genomes again raises the question of how many large $(>200 \mathrm{~nm})$ 
phages are excluded by size fractionation (see section CultureDependent Methods).

Unusual, composite phages have also been identified to play a role within the gut. Bacterial strain Enterococcus faecalis V583 contains multiple prophage elements, two of which combine to form a lytic phage particle (Duerkop et al., 2012). One of these distinct prophages encodes the structural proteins, while the other contains necessary infection elements, and both are essential for lytic infection. The resultant phage appears to give E. faecalis V583 a competitive advantage over related strains when colonizing the gut (Duerkop et al., 2012) (see discussion in section Phage-Host Population Dynamics). While being of interest as a stand-alone case, this also has implications for future metagenomic studies when partial prophage-like sequences are found.

\section{Longitudinal Distribution of Phages in the Gastrointestinal Tract}

The location within the gut also influences the phageome (Figure 2). Following the stomach, the proximal gut provides the most hostile environment for bacteria, a microaerophilic environment with a low $\mathrm{pH}$ and antimicrobial peptides which enriches bacteria such as Lactobacillaceae and Enterobacteriaceae. The distal gut is the opposite, an anaerobic environment, with a higher $\mathrm{pH}$ and reduced concentration of antimicrobial peptides, allowing bacteria such as Bacteroidaceae, Prevotellaceae and Ruminococcaceae to thrive (Donaldson et al., 2016). This longitudinal gradient causes a steady increase in bacterial load and diversity from the proximal to the distal gut, and it may be expected that the phage distribution would also follow this longitudinal axis. In primate models, it has been shown that both the relative abundance of phages and the composition of the phageome varies depending on site within the gastrointestinal tract (Zoetendal et al., 2012; Zhao et al., 2019). Furthermore, a study in pigs found a higher abundance of phages in the ileum, and hypothesised that the nutrient-limited environment may prevent bacterial phage resistance and promote pseudolysogeny, allowing phage proliferation (Looft et al., 2014). The density of phage populations also varies cross-sectionally through the mucus layers of the gut (Barr et al., 2013a), the implications of this are discussed further in section Interactions With the Immune System. These differences in phage communities hint at the different roles and functions of phages throughout the gastrointestinal tract, that require greater understanding to use phages in the future as markers of gut health or as treatments to improve gut function. In Figure $\mathbf{2}$ we have displayed the varying composition of the phageome throughout the digestive tract, where the majority of the knowledge arises from faecal samples.

\section{Impact of Diet on the Phageome}

A well-characterised influence on the gut microbiome is diet, therefore it can be assumed that diet will impact the phageome too (Singh et al., 2017). This has been observed in studies of high fat diet (HFD) in mouse models, wherein both the microbial and viral gut communities were affected (Howe et al., 2016; Schulfer et al., 2020). These changes to the phageome during a long-term
HFD included an increased abundance of Microviridae, while Siphoviridae abundance decreased (Schulfer et al., 2020). As breast milk is high in fat, this may provide some insight into why previous studies have found that infant gut phageomes move towards Microviridae-dominated communities (see section Development of the Phageome) (Figure 3). The change of diet in infancy, to weaning and solid foods was shown to alter gut phage composition and diversity in pandas (Guo et al., 2020), suggesting the change from infant to adult phageome is in part influenced by dietary factors. Malnutrition in infancy was also found to affect the gut phageome (Reyes et al., 2015). The microbiome and phageome developed sequentially, but the phageome was disrupted in malnourished infants (Reyes et al., 2015). Phage populations could not be recovered, despite the use of dietary supplements, suggesting that infancy is a key time in the development of the gut phageome (Reyes et al., 2015). Further studies have identified that the phageome is not only distinct in malnourished children, but in fact these phages may play a pathophysiological role (Mirzaei et al., 2020). In vitro experiments indicated phages from a stunted child caused microbiota shifts to a disease associated state in an age-related manner (Mirzaei et al., 2020). The study suggested there is a time window where the phageome and microbiome of children less than 23 months is amenable, before the malnutrition-associated community becomes fixed. As malnutrition and stunting of growth is a major cause of mortality in infants, this finding is of great importance.

Once the phageome is established it is less influenced by diet, but increasing fat does seem to have an impact. Increased fat leads similar shifts in the gut phageome, however there was still great inter-individual variation (Minot et al., 2011). This suggests that the phageome is not acquired through diet, but that the diet shapes existing communities (Minot et al., 2011). In addition, consumption of various foods can influence prophage induction in the resident gut microbiota (Kennedy et al., 1984; Jofre et al., 1995; Méndez et al., 2004).

Numerous studies have identified food products that are a rich source of bacteriophages, yet there is little evidence if or how they influence the phageome. Coliphage can be found in a variety of food sources, and are used as an indicator of faecal contamination (Kennedy et al., 1984; Kennedy et al., 1986; Jofre et al., 1995; Méndez et al., 2004). Gut viruses include eukaryotic and plant viruses that arrive in the gut via the oral route (Liu et al., 2013), which have been demonstrated to make up a high proportion of RNA viruses detected in the gut (Zhang et al., 2005). There is also evidence of bacteriophages surviving food decontamination processes, such as fermented foods; including soy beans (Chukeatirote et al., 2018), sauerkraut (Yoon et al., 2002; Lu et al., 2003), cheese (McIntyre et al., 1991; Quiberoni et al., 2006), yoghurt (Ma et al., 2014; Ma et al., 2015), fermented vegetables (Lu et al., 2003; Kleppen et al., 2012), fermented meats (Trevors et al., 1984), drinking water (Armon et al., 1997) and heat-stable phages surviving dairy product pasteurisation (Suarez et al., 2002; Murphy et al., 2014). Furthermore, in recent years, there has been a rise in the consumption of unpasteurised milk, unfortunately linked to a 
number of bacterial diseases (Lucey, 2015). Unpasteurised milk has been demonstrated to be an abundant source of new bacteriophages, containing up to $10^{4}$ bacteriophages per $\mathrm{mL}$ of product (Marco et al., 2012). To date, no studies have examined the effect of dietary phage intake to understand the impact of normal dietary intake on the phageome and wider microbiome.

Bacteriophages are currently being used to make food safer and have been used as indicators for the virological safety of food (Allwood et al., 2004), as alternatives to antibiotics in animal husbandry (Cheng et al., 2014; Gigante and Atterbury, 2019), in food decontamination (Moye et al., 2018), to increase yield or quality (Zhao et al., 2012), and to prevent spoiling (Sillankorva et al., 2012). Table 4 displays a list of bacteriophage products approved for use in food manufacturing. As the use of these phage products become increasingly more common, their abundance in the human diet is likely to increase; the impact on the human gut microbiome is currently unknown. Drinking water is another potential source of phages, regardless of high quality water treatment systems and microbiologically safe drinking water, viruses survive the process (Armon et al., 1997). While large scale changes due to dietary consumption of phages appear transient, there is the potential for a variety of viruses to enter the digestive system and influence the human host.

The human gut is an abundant source of potential host bacteria, which should allow many dietary phages to thrive. Equally there are a number of factors that may prevent colonisation by dietary phages, including phage-specific antibodies (Mirzaei and Maurice, 2017), eluded to by a higher abundance and diversity within the phageome of patients with immune disorders (Norman et al., 2015; Perez-Brocal et al., 2015). Phage colonisation may be further hampered by various exclusion strategies by host strains (Rossmann et al., 2015) and phage competition (Reyes et al., 2013). It has been shown that orally delivered phages can survive the stomach to be detected in the faeces, while some phage treatments reduce target bacteria (Mai et al., 2015), not all phage-host systems are equal and some phages fail to proliferate (Sarker et al., 2016). With GI tract phageome studies limited in scope and number, the lack of direct supporting evidence for dietary phages to shape the phageome and microbiome does not mean that they play no role. Instead, it is another overlooked aspect of the human gut microbiome, particularly in the first two formative years.

\section{Impact of Medical Interventions on the Phageome}

Medical procedures and treatments can have an effect on the gut phageome, including the well-documented impact of antibiotics. The effect of broad spectrum antibiotics on the gut bacterial community is non-targeted killing, leading to a dysbiotic state (Jernberg et al., 2010), which may in turn affect the phageome. In rhesus monkeys there has been an observed decrease in viral abundance and diversity following antibiotic treatment ( $\mathrm{Li}$ et al., 2019), but no detectable change in the gut phageome was found in human studies of long-term antibiotic use (Abeles et al., 2015). It may be that disturbances in the gut viral community caused by antibiotics are rapid, and during long-term exposure the community re-adjusts and stabilises. In the short-term, prophage induction has frequently been demonstrated upon antibiotic exposure due to host DNA damage and initiation of the bacterial SOS response, in turn activating prophages and potentially increasing the likelihood of horizontal gene transfer (Maiques et al., 2006; Allen et al., 2011; Lin and Lin, 2019), further discussed in section Virus-Mediated Genetic Exchange. This has consequences for subsequent antibiotic usage, if a reservoir of phage-encoded antibiotic resistance genes is established in the gut (Modi et al., 2013; Abeles et al., 2015).

Faecal microbiota transplant (FMT) is the transfer of faecal material from a healthy donor to an individual with gastrointestinal disease with the intention to restore a healthy gut microbiome. It has been shown that the phage component is of high importance in FMT, able to improve efficacy (Bojanova and Bordenstein, 2016; Zuo et al., 2018) and remains effective without the bacterial fraction of FMT (Ott et al., 2017).

TABLE 4 | Phage products approved for use in food manufacturing. These phage products have the opportunity to enter the Gl tract and impact the gut phageome.

\begin{tabular}{|c|c|c|}
\hline Manufacturer & Product & Applications \\
\hline \multirow[t]{4}{*}{ Intralyx Inc. (MD, USA) } & ListShield $^{\mathrm{TM}}$ & Targets Listeria monocytogenes contamination in foods and food processing facilities. \\
\hline & EcoShield ${ }^{\mathrm{TM}}$ & Targets Escherichia coli, and Shiga toxin producing E. coli in particular, including O157:H7 STEC. \\
\hline & SalmoFresh PX ${ }^{\mathrm{TM}}$ & $\begin{array}{l}\text { Targets contamination with selected, highly pathogenic Salmonella-serotypes in foods and food processing } \\
\text { facilities. }\end{array}$ \\
\hline & ShigaShield ${ }^{\mathrm{TM}}$ & Targets contamination with Shigella spp. in foods and food processing facilities. \\
\hline APS Biocontrol (Dundee, UK) & Biolyse ${ }^{\circledR}$-PB & $\begin{array}{l}\text { Targets bacteria that cause soft rot on potatoes mainly, Erwinia spp., Pectobacterium spp., and Pseudomonas } \\
\text { spp. }\end{array}$ \\
\hline \multirow[t]{2}{*}{ Proteon Pharmaceitocals } & BAFASAL ${ }^{\circledR}$ & Targets human-pathogenic Salmonella spp. in poultry farming. \\
\hline & BAFADOR $^{\circledR}$ & Targets Pseudomonas spp. and Aeromonas spp. in commercial aquaculture \\
\hline Elanco (IN, USA) & Finalyse ${ }^{\mathrm{TM}}$ & Pre-slaughter hide wash applied to live cattle, targeting E. coli O157:H7 \\
\hline \multirow[t]{3}{*}{$\begin{array}{l}\text { Micreos Food Safety, (The } \\
\text { Hague, NL) }\end{array}$} & $\begin{array}{l}\text { PhageGuard } \\
\text { Listex }^{T M}\end{array}$ & $\begin{array}{l}\text { Surface treatment targeting Listeria monocytogenes on a number of food products such as, meat, fish, cheese } \\
\text { and frozen vegetables. }\end{array}$ \\
\hline & PhageGuard $S^{T M}$ & Targets Salmonella spp. on fresh poultry. \\
\hline & PhageGuard $E^{\mathrm{TM}}$ & Targets E. coli O157 on beef carcuses. \\
\hline OmniLytics (UT, USA) & Agriphage $^{\mathrm{TM}}$ & $\begin{array}{l}\text { Targets Xanthomonas campestris. Xanthomonas vesicatoria and Pseudomonas syringae to prevent bacterial spots } \\
\text { tomatoes and peppers. }\end{array}$ \\
\hline
\end{tabular}


The mechanism behind this has not yet been established; it may be due to an alteration of the patient's own phageome or the predatory action of the donated phages. A number of studies have looked at the potential of faecal virome transplants (FVT) from one individual to another, which could assist recovery after antibiotic-induced dysbiosis (Draper et al., 2019).

In contrast with the microbiome, there has been limited success in defining a core phageome. The phageome is highly specific to an individual and its resilience to change over time raises interesting questions. The phageome appears to remain relatively constant, while we know the microbiome fluctuates over time and with environmental factors. But are there as yet undetected effects on the phageome? Do phage populations remain dormant for a long time, persisting in host populations, or is their host range wide enough that they continue to encounter hosts regardless of fluctuations in bacterial populations? We know that phages are consumed in the diet, and it is assumed with the survival of other viruses, phages also survive the human gastrointestinal tract. If this is the case, are phages able to colonize the gut once they reach it? As seen with FMT, this appears to be possible as phages seem to be the key to success of this treatment. However, the stability of the phageome within an individual suggests that no such invasion and colonisation occurs. Answering this myriad of questions is hampered by the sampling method used to study the phageome: metagenome sequencing of faeces (see section Metagenomics). Measuring phage activity throughout the GI tract over a time course would help to elucidate fluctuations in the phageome at sites where it could impact human health and gut function. As this is still an emerging field, we hope with greater investigation the phageome will reach a level of understanding we see for the microbiome.

\section{PHAGEOME, GUT BACTERIA, AND HUMAN HOST INTERACTIONS}

Given that most phages infect and eventually lyse specific bacterial hosts, the gut bacterial community shapes the phage community, as much as it is shaped by phages. Phage-host interactions occur in a number of context-dependent dynamics throughout the gastrointestinal tract, playing roles in the cycling of nutrients, community function and transfer of genetic material. The role of the phageome is seen primarily through its influence on the function of the bacterial community, however there are further consequences of their presence in the gut, including on human immune responses, impacts on human health and gut-associated bacterial biofilms.

\section{Phage-Host Population Dynamics}

In the human gut, the phageome is thought to affect succession and colonisation events (Lozupone et al., 2012; De Sordi et al., 2019; Shkoporov et al., 2019), it is well known that phage contribute to feedback loops and predation which influence the dominance and diversity within a microbial population. Broadly speaking, there are three main dynamics under which the phageome will be able to alter the gut microbiome: Red Queen dynamics [continual evolution of defence and counter-defence measures (Table 5)] (Van Valen, 1973), kill-the-winner (lysis of common genotypes, preventing dominance) (Maslov and Sneppen, 2017), and piggyback-the-winner (lysogeny allowing phage and bacteria to stably co-exist) (Weitz and Dushoff, 2007).

On the mucosal surfaces of the gut, the bacterial population is spatially structured through the surrounding mucus. The lifestyle of phages is dependent on host availability: less available hosts, lytic predation takes place, and otherwise, lysogeny is the key mode of phage lifestyle (Figure 4). It is theorised this kill-thewinner strategy promotes elimination of potential pathogens deep in mucus layers, while a lysogenic piggyback-the-winner strategy provides an advantage for bacterial commensals against niche invasion (Silveira and Rohwer, 2016).

As a consequence of these changes and alterations in phage dynamics and prevalence in the human gut, it is possible that no specific genotypes maintain dominance, but are constantly superseded by functionally equivalent strains, maintaining stable metabolic potential and taxonomic diversity. This functional redundancy is known to be favoured in the bacterial microbiome (Ley et al., 2006), allowing more resilience and less impact of dysbiotic perturbations (Vieira-Silva et al., 2016).

\section{Virus-Mediated Genetic Exchange}

Bacteriophages are able to influence evolution and diversification of bacterial communities through horizontal gene transfer (Wommack and Colwell, 2000; Koskella and Brockhurst, 2014). As previously discussed, lysogenic phage and prophage dominate the human gut (Breitbart et al., 2003; Reyes et al., 2010; Minot et al., 2011). Lysogenic conversion of bacterial phenotype has been shown to contribute to $5 \%$ of the conserved microbiome function in the human gut, indicating these prophage exert a strong influence over the microbial function in this environment (Qin et al., 2010). The effects of genetic exchange are well-studied in pathogenic species, but have also been demonstrated in human gut commensals such as Lactobacillus gasseri (Baugher et al., 2014).

Further to the impact on the bacterial hosts, viral genetic exchange from the gut microbiome has wider implications for the mammalian host, with viral integrases being shown to mediate chromosomal integration in human cells (Groth et al., 2000). The larger impact of this has yet to be explored.

Due to the global threat of rising antimicrobial resistance, the contribution of viral genetic exchange has been studied in depth (Balcazar, 2014). It has been theorised that bacteriophage could be the missing link, transferring antimicrobial resistance genes (ARGs) from the environment into the human microbiome, as well as between bacteria within the microbiome (Muniesa et al., 2013; Quiros et al., 2014). Phages may contribute more to the dissemination of these genes compared to plasmids and chromosomal elements, both over time-scale and wider spaces (Debroas and Siguret, 2019). This will be especially important in the densely populated human gut, where numerous transduction events potentially occur at any given moment. In a mouse study, after exposure to antibiotics, there was an enrichment of phageencoded genes associated with antibiotic resistance and/or antibiotic mitigation in faecal samples (Modi et al., 2013). 
TABLE 5 | Summary of bacteria-encoded phage defence mechanisms.

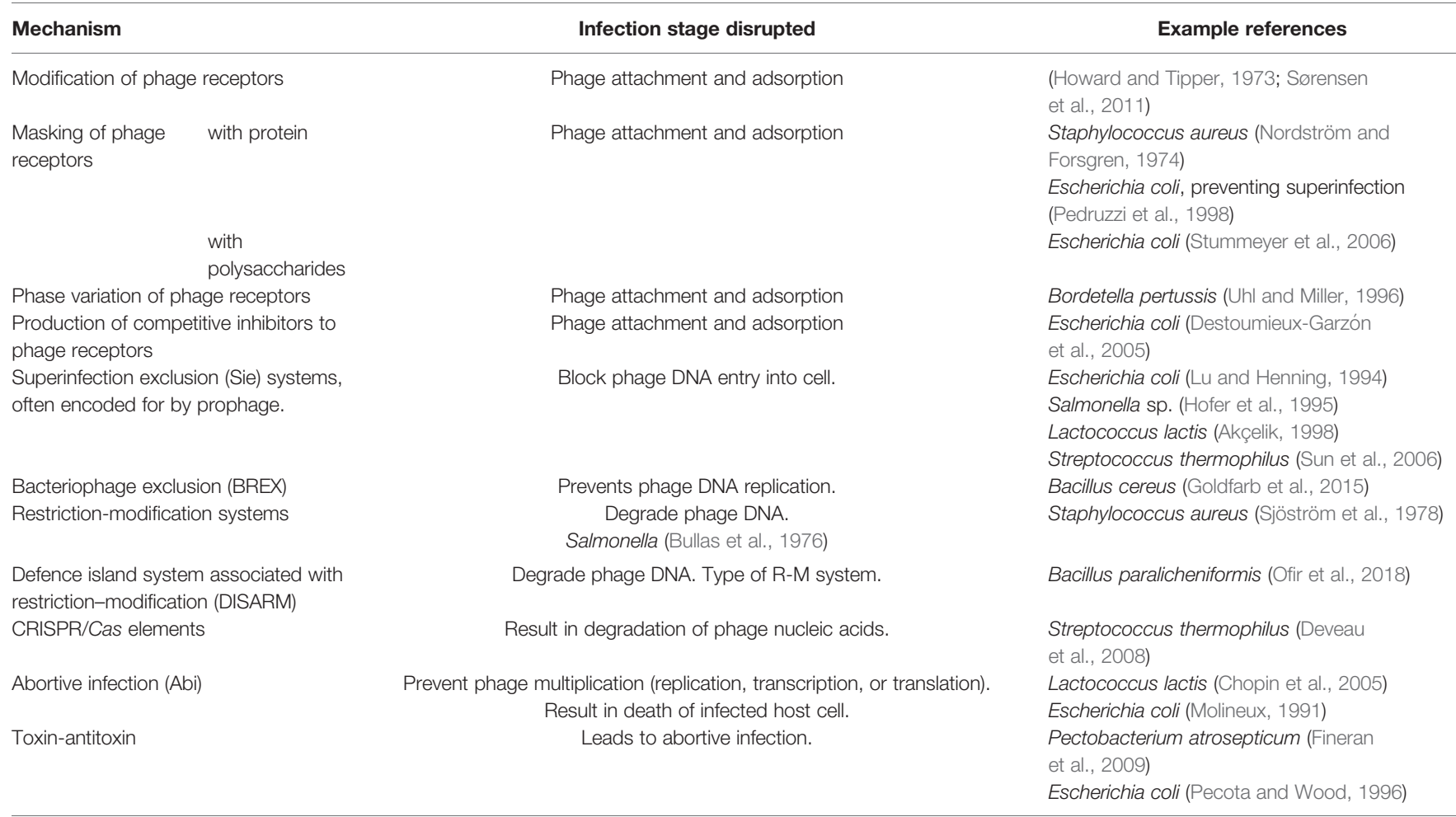

This was not only to the applied antibiotic, but also to other classes. Critically, these phage-encoded genes were shown to be functional through transfecting antibiotic naïve mice (Modi et al., 2013). On a cautionary note, while these phages were able to transfer antibiotic resistance between mice, the true rate of antibiotic resistance encoded by phages is much lower than predicted by bioinformatic methods (Enault et al., 2017). Despite this, phages can facilitate the release and exchange of plasmidencoded ARGs when they lyse their hosts.

\section{Cycling Key Nutrients}

Nutrient cycling by phages is well-characterised in environmental systems, such as marine and soil ecosystems. The "viral shunt" explains how carbon and other intracellular nutrients are released upon lysis of host cells into the extracellular milieu. (Wilhelm and Suttle, 1999). The human gut has significantly more available nutrients compared to marine and soil systems, leading to the extremely high cell densities, resulting in more competition for available nutrients. Examples of key nutrients which are limited for the gut microbiota are iron and vitamin $\mathrm{B}_{12}$ (cobalamin) (Deriu et al., 2013; Degnan et al., 2014). It has been proposed that gut viruses play a role in alleviating this competition, limiting growth, regulating community stability and nutrient cycling, as observed in other systems (Lozupone et al., 2012). Due to the high degree of interindividual gut community variation, it is difficult to pinpoint how the phageome alters nutrients in the gut or is itself altered by incoming nutrients from the human host diet (see section Impact of Diet on the Phageome) (Minot et al., 2011; David et al., 2014).

\section{Interactions With the Immune System}

While phage interactions with their bacterial hosts are of vital importance, phage interactions with the human host are also paramount. There is evidence that the phageome is able to influence both the innate and adaptive system, playing a defensive role at the gut mucosa (Barr et al., 2013b; Barr et al., 2015). It has been shown that immune stimulation can be caused by a number of phage proteins (Majewska et al., 2015; Miernikiewicz et al., 2016), and possibly cause a low-grade inflammation in the intestines, without any clinical symptoms (Norman et al., 2014). However, the correlation of phage in FMT with positive outcomes in inflammatory bowel disease (Gogokhia et al., 2019) [see section Inflammatory Bowel Disease (IBD)] suggests a more complex relationship between the phageome and individual human health.

Phage proteins, not involved in the attachment to the bacterial host, have the ability to instead attach to the gut mucosa. These adhesins have been identified on the capsid, collar whiskers and tail shaft of the phages, and possess immunoglobulin-like (Ig-like) domains (Fraser et al., 2007; Barr et al., 2013a). Along with these Ig-like domains, regions of hypervariability in the virus tail fibres help to mask phages from the immune system (Doulatov et al., 2004; Minot et al., 2012). The Ig-like domains additionally function to bind the phages to the mucus, allowing phages to form a defensive barrier over mucosal membranes (Figure 4). This barrier is evidence of a symbiotic coevolution between phages and the human host, by enabling phages to come into contact with bacterial prey and regulating the bacterial populations at this surface, benefiting 


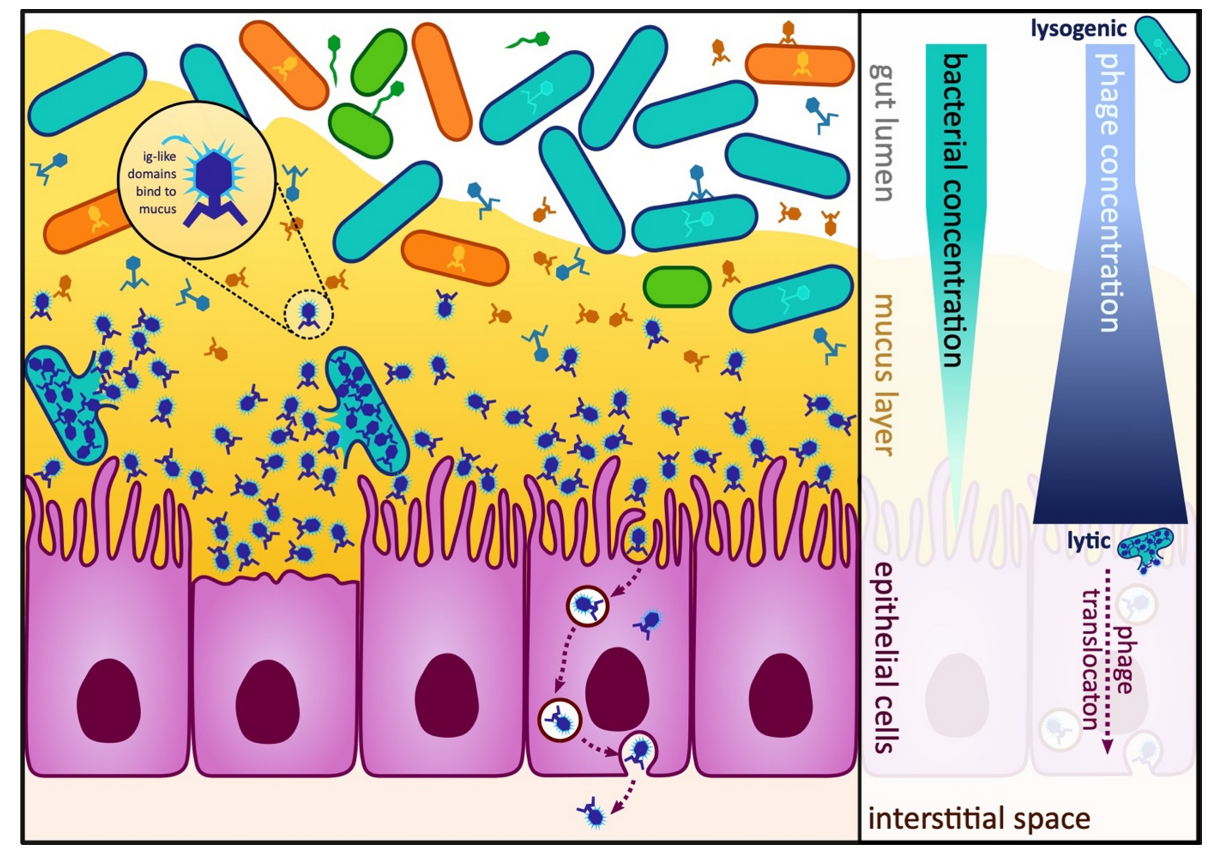

FIGURE 4 | Diagram of the gut and bacterial and phage concentrations in the lumen, mucosa and epithelial cells. In the human gut, multiple different host-prey dynamics are theorised to occur dependent on the proximity to the gut mucosa. At the top of the mucosa, viruses take a lysogenic strategy or "piggyback-thewinner" as hosts are abundant here. Deeper within the mucosa, the viruses switch to a lytic or "kill-the-winner" strategy as at this point the bacteria are less abundant (Silveira and Rohwer, 2016). (Right) Some phages encode Ig-like domains that allow them to bind to the mucosa and evade the immune system, (Left) Phages can undergo transcytosis and be engulfed and transported through epithelial cells.

human health (Barr et al., 2013a). Bacteriophages have been shown to be actively transported across the gut epithelium, by transcytosis to variable degrees (Bruttin and Brussow, 2005; Gorski et al., 2006; Nguyen et al., 2017) (Figure 4), meaning there is more opportunity for interaction with immune cells outside of the gut.

It is clear from the summative research conducted that phages, in addition to affecting their bacterial hosts, impact the health of the human or mammalian hosts in which they reside. This could have an important impact for numerous diseases, as discussed later in section, Role of Bacteriophages in Human Disease.

\section{Phage Behaviour Within a Biofilm Environment}

There is evidence to suggest that the majority of microbial life within the gastrointestinal tract exists as a biofilm, a distinct lifestyle to planktonic bacteria (Costerton et al., 1987; Macfarlane and Dillon, 2007; Hussain et al., 2020). As the bacterial host behaves significantly differently within a biofilm-dominated environment, it is important to consider how this will impact phage behaviour which is predominantly investigated in planktonic in vitro settings.

The biofilm within the gut lumen performs multiple functions, but one of the most important is acting as a physical barrier to the mucosa. Commensal bacteria are able to form a protective coating over the mucosa which will prevent pathogenic bacteria penetrating to the epithelial cell layer (Iacob et al., 2019). However, pathogenic bacteria may invade the biofilm, lay dormant and benefit from the surrounding cells by acquiring ARGs and evading host defences. This may then lead to a disease state when the cells exit dormancy (Tytgat et al., 2019).

The self-produced extracellular matrix (ECM) of a biofilm is recognised as a protective coating of cells, preventing diffusion of small molecules through the bacterial population (Flemming and Wingender, 2010). Numerous phages have been shown to encode depolymerases which are able to degrade ECM, allowing them to make contact with host cell receptors (Hughes et al., 1998). As these are widespread, it appears to indicate that the ECM is a major barrier to phage infection (Pires et al., 2016).

However, phages are also able to promote the formation of a thicker biofilm, which as previously stated can be associated with disease. Quorum sensing, a communication system within biofilms, is able to induce temperate phages. These phages are then able to contribute to HGT within the biofilm, as well as bacterial host evolution and adaptation through insertional activation of genes (Rossmann et al., 2015; Davies et al., 2016). Phages can also provide physical additions to the ECM, through both the presence of phage particles, and released cellular contents after lysis, particularly eDNA (Rice et al., 2009; Schuch and Fischetti, 2009; Carrolo et al., 2010; Wang et al., 2010; Shen et al., 2018). 
The presence of biofilm in the gut lumen is a double-edged sword; it protects the human host but has the potential to harbour pathogens and enhance disease, as seen in inflammatory bowel disease (Swidsinski et al., 2005). Equally, phages can both promote or disrupt this biofilm state. Therefore it is difficult to say what the wider impact of phage populations are in this case. These interactions should be considered before the application of phage therapy, where there may be unintended consequences of disrupting "healthy" biofilms or enhancing "diseased" biofilms.

Understanding how a single phage interacts with its infection host is quite different to how a population of phage interacts with not only bacteria but human cells, fungi and archaea which may dwell in the gut. It has been shown in vitro that a bacteriophage can seemingly impact on Candida albicans, a dimorphic fungus that can be found in the gut (Nazik et al., 2017). In this section, we explained how phages may prime the immune system, but to our knowledge there is no investigation into interactions with other non-bacterial cells. As gut communities become more widely studied, this may soon be elucidated. Having a controlled system is beneficial as it allows determination of these minute interactions, but it is no use if it does not reflect the in vivo situation. One in vitro system which could be utilised complex interaction studies, is a gut fermentation model seeded with faecal samples, currently used in the study of phage therapy for C. difficile (Nale et al., 2018). By expanding to a more "wideangle lens" of phage interactions, would open the possibility to discover important off-target effects in microbial and human cells.

\section{ROLE OF BACTERIOPHAGES IN HUMAN DISEASE}

In recent years, our understanding of the intestinal microbiome has increased to encompass the ways in which it impacts on human health and disease. The majority of this research has focussed on the bacterial populations, yet the phageome is also expected to have a distinct role in shaping the gut environment (Table 6). Even plant viruses which enter through the diet (section Impact on Diet on the Phageome), such as Pepper mild mottle virus, elicit an immune response in humans. This suggests that viruses' effects are not limited to infective host range (Colson et al., 2010).

Phages can have both direct and indirect impacts upon the gut environment, leading to systemic consequences for human health. Phage predation of gut bacteria, particularly those that have protective roles in human health, can lead to dysbiosis and disease.

For example, the diseased gut environment can be inflammatory, leading to prophage induction in Salmonella, causing bacterial lysis and initiating gut dysbiosis (Diard et al., 2017). As previously discussed in section Interactions With the Immune System, phages can also control populations of invasive bacteria in the gut and have a role maintaining the intestinal barrier function (Barr et al., 2013a). Conversely, gut phages can also directly increase intestinal permeability, leading to translocation of bacteria and bacterial products from the gut into the bloodstream, which contributes to a chronic inflammatory response (Tetz et al., 2017).

\section{Inflammatory Bowel Disease (IBD)}

Crohn's disease (CD) and ulcerative colitis (UC) are the two major types of IBD. CD is characterised by chronic inflammation throughout the gastrointestinal tract, most commonly impacting the ileum and colon, whereas UC involves inflammation and ulceration limited specifically to the colon and rectum. CD can affect the entirety of the intestinal wall, leading to the development of complications such as abscesses, fistulas and strictures, whereas UC affects the inner intestinal lining to cause crypt abscesses and cryptitis (Khor et al., 2011). Understanding of IBD pathology has shown that genetic predisposition interacts with the gut microbiota and environmental cues, leading to the development of disease. On the surface IBD shows no obvious direct links to the gut phageome, however we are only beginning to understand how phages are an intrinsic part of the gut community and how they are linked to IBD.

Individuals with CD may show an altered phageome compared to healthy control subjects. Epifluorescent microscopy of biopsies from both ulcerated and non-ulcerated tissues, from $\mathrm{CD}$ and control patients, showed over 10-fold higher VLPs in individuals with CD, than in the control

TABLE 6 | Summary of changes to the gut phageome in different disease states.

\begin{tabular}{|c|c|c|c|}
\hline Disease & Bacteriophage Richness & Bacteriophage Diversity & Reference \\
\hline \multirow[t]{5}{*}{ Crohn's disease } & + & + & (Lepage et al., 2008) \\
\hline & + & + & (Perez-Brocal et al., 2015) \\
\hline & & + & (Norman et al., 2015) \\
\hline & - & & (Fernandes et al., 2019) \\
\hline & + & & (Wagner et al., 2013) \\
\hline \multirow[t]{3}{*}{ Ulcerative colitis } & + & & (Lepage et al., 2008) \\
\hline & + & - & (Zuo et al., 2019) \\
\hline & + & & (Gogokhia et al., 2019) \\
\hline \multirow[t]{2}{*}{ Type 1 diabetes } & & - & (Zhao et al., 2017) \\
\hline & & + & (Tetz et al., 2019) \\
\hline Type 2 diabetes & + & & (Ma et al., 2018) \\
\hline Human Immuno Virus & + & & (Li et al., 2012) \\
\hline \multirow[t]{2}{*}{ Cardiovascular disease } & $+/-$ & & (Jie et al., 2017) \\
\hline & + & & (Han et al., 2018) \\
\hline
\end{tabular}


biopsies (Lepage et al., 2008). These VLPs were consistent with Siphoviridae, Myoviridae and Podoviridae morphotypes, and were detected more frequently in non-ulcerated mucosa, when compared to ulcerated tissues from the same individual (Lepage et al., 2008). The diversity of the gut phageome is higher in CD patients compared to healthy controls, and again higher in newonset compared to active CD patients (Norman et al., 2015; Perez-Brocal et al., 2015). In contrast to these studies, (Clooney et al. (2019) found little change in diversity of phage populations. However, they did observe a reduction in health-associated virulent phages, with an increase in unique temperate phage populations which became predominantly lytic rather than lysogenic (Clooney et al., 2019). Within UC patients, there were subtle changes in the phageome during a disease flare up or remission state. The authors highlighted again that it is difficult to elucidate if these changes are initiated by the bacterial or phage populations. Regardless, these viral markers may be involved in mediating an inflammatory response, be induced in response to the CD patients intestinal inflammation, or potentially both.

In addition to a general increase in the abundance of phages seen in patients with $\mathrm{CD}$, the emergence of dominant phage families has been studied in the gut viral community. Caudovirales are the most dominant phage family present in CD patient samples (Wagner et al., 2013; Norman et al., 2015; Fernandes et al., 2019). An inverse correlation was observed between Caudovirales richness and diversity compared to bacterial richness and diversity, indicating that the increase in Caudovirales is not linked to host numbers in CD (Norman et al., 2015), but rather that phage may be the driving force behind fluctuations in disease. A decreased abundance of Microviridae has also been reported in CD patient samples (Fernandes et al., 2019).

Bacteroides is commonly identified as the host of these phages, with the two most prominent phages identified infecting Bacteroides fragilis, a bacterium associated with healthy gut function (Kang et al., 2010; Wagner et al., 2013). As Bacteroides is the dominant bacterial population in $\mathrm{CD}$, the role of these phages may warrant further investigation (Murphy et al., 2008; Andoh et al., 2011). As the levels of both phage and host increase, this could indicate a lysogenic relationship or blooming of the host in CD. Faecalibacterium prausnitzii also plays a key role in $\mathrm{CD}$ and mediates anti-inflammatory effects; a reduction in $F$. prausnitzii has been associated with the onset of $C D$ (Martinez-Medina et al., 2006). Metagenomic analysis shows patients with IBD have increased numbers of $F$. prausnitzii phages (Cornuault et al., 2018), suggestive of a predatory phage function. Experimental administration of a phage cocktail against pathogenic Enterobacteriaceae, Streptococcaceae and Staphylococcaeceae, led to an off-target decrease in gut Faecalibacterium abundance, considered indicative of inflammation and a change in gut permeability (Tetz et al., 2017). Potentially, these off-target phage effects evidence that our knowledge of phage-host ranges may not be as narrow as we expect, and could have a downstream impact on symbiotic gut bacteria association with healthy gut function (Tetz et al., 2017).
It is often assumed that phage therapy minimally disrupts a patient's microbiome, however it may lead to unforeseen downstream effects in the gut.

In parallel to $\mathrm{CD}$, the gut mucosa of individuals with $\mathrm{UC}$ contains an increased abundance of Caudovirales compared to healthy subjects, though unlike in $\mathrm{CD}$, a lower phage diversity is seen (Zuo et al., 2019). Mouse studies associated this with intestinal inflammation and a more severe disease presentation (Gogokhia et al., 2019). A specific increase in Escherichia, Streptococcus and Enterobacteria phages is seen in UC patients (Zuo et al., 2019). Previous research has found the abundance of these bacteria to be lower in those with UC when compared to healthy controls, suggesting predatory phage or prophage induction, rather than the phageome mirroring host bacteria abundance (Michail et al., 2012). Furthermore, phage functions associated with host bacterial fitness and pathogenicity were higher in the UC gut mucosa (Zuo et al., 2019). In mouse studies, those with colitis have more phages which are not associated with their bacterial hosts, suggesting a state of intestinal dysbiosis (Duerkop et al., 2018). In a number of studies with UC patients, inflammation of gut mucosa translated to an increase in viruses, particularly Caudovirales phages, when compared with the noninflamed mucosa, indicating that Caudovirales could play a direct role in inducing inflammation in the gut (Lepage et al., 2008; Zuo et al., 2019). Enrichment of gut phages appears to be specific to the UC disease state, as healthy controls showed an enrichment of eukaryotic viruses (Zuo et al., 2019).

Gogokhia et al. (2019) found that intestinal Caudovirales correlated with the success of FMTs in humans with UC. UC patients that responded well to FMT had a lower abundance of Caudovirales before FMT than after treatment, suggesting that the intestinal phage community could worsen disease (Gogokhia et al., 2019). This has implications for the potential use of FMT therapy and clinicians should consider the impact of phages on patient outcomes.

Comparing phage populations of CD and UC patients shows a significant difference in the composition of the gut virome, suggesting that even within IBD the phageome can be influenced by environmental changes specific to each disease (Norman et al., 2015). Taken together, this evidence suggests that phages play a role in IBD, particularly within UC and CD. However, the difficulty in moving from correlation to causation in the disease pathology warrants further research into the role of phages as drivers of human diseases.

\section{Diabetes}

Diabetes is a group of diseases characterised by hyperglycemia, caused by a defect in the action of insulin, the secretion of insulin, or a combination of both (American Diabetes Association, 2009). Type 1 diabetes (T1D) is caused by the autoimmune destruction of pancreatic b-cells, whereas Type 2 diabetes (T2D) is a result of insulin resistance (American Diabetes Association, 2009). The gut environment has been associated with the onset of both T1D and T2D, as environmental factors within the gut are thought to act as triggers of disease development. 
In patients with $\mathrm{T} 1 \mathrm{D}$ a comparison of stool samples to healthy controls showed that the evenness of two major phage groups, Myoviridae and Podoviridae, was lower, suggesting that the distribution of gut phage is disturbed in individuals with T1D (Zuo et al., 2019). Amyloid-producing E. coli present in the gut have been linked to the development of autoantibodies contributing to T1D (Tetz et al., 2019). Diversity of intestinal E. coli phages was found to be significantly higher in T1D patients than in control individuals, with almost all the identified E. coli phages being lysogenic (Tetz et al., 2019). The ratio of phages to $E$. coli was also shown to increase in those with T1D, suggesting continual induction of prophages, or E. coli harbouring prophages may have a fitness advantage in T1D (Tetz et al., 2019). Bacteroides dorei has also been found at higher abundance in individuals with T1D and those at risk of developing islet autoimmunity (Leonard et al., 2014). There are a number of methylase genes present in the genomes of $B$. dorei temperate phages, which raises questions about the impact of methylation and prophages on the development of islet autoimmunity (Leonard et al., 2014). In samples from T1D patients, CrAssphage were shown to correlate with B. dorei, but no other Bacteroides (Cinek et al., 2017). However, no significant relationship between the presence of CrAssphage and the onset of islet autoimmunity was identified (Cinek et al., 2017).

Compared to healthy control samples, significantly more phages were present in the gut of individuals with T2D, which showed an increased abundance of intestinal Podoviridae predicted to infect Escherichia and Clostridium (Breitbart et al., 2018). As Clostridium spp. and E. coli have been found to be present in higher numbers in T2D patients, this could suggest that these are temperate phages which proliferate alongside their host bacteria in accordance with the development of T2D (Qin et al., 2012). T2D patients' gut phages carried a large number of genes which could impact the human host (Ma et al., 2018), suggesting that phages themselves could drive the pathogenesis of human diseases.

Interestingly it has been demonstrated that fecal virome transplant (FVT) can alleviate obesity and T2D in a mouse model, significantly altering both the bacterial and viral components of the gut microbiota (Rasmussen et al., 2020). FVT in mice alleviated some effects of a high-fat diet and normalised blood glucose tolerance, which was not observed in control mice (Rasmussen et al., 2020). It has also been suggested that both Caudovirales richness and the overall viral diversity in donor faeces can influence the outcome of FVT in T2D, warranting further investigation (Zuo et al., 2018; Park et al., 2019).

Despite the research to date, the question remains whether gut phages influence the onset and development of T1D and $\mathrm{T} 2 \mathrm{D}$, or whether they act as disease biomarkers, revealing the effects of diabetes on the gut microbiome. The role of temperate phages in human disease is currently unknown and it is yet to be discovered if prophages are induced in the gut environment and drive the development of T1D or T2D, or if prophage induction is a response to autoimmunity.

\section{Human Immunodeficiency Virus (HIV)}

$\mathrm{HIV}$ is a highly complex disease with wide-ranging systemic consequences for the patient, even with the use of antiretrovirals. The interaction of the gut, blood, and immune system complicates investigation, but it appears that phages may predict disease progression and influence responses to therapy. HIV infections lead to a depletion in CD4+ T cells, rendering the body vulnerable to infection and disease. The advanced stages of $\mathrm{HIV}$ infection are referred to as acquired immunodeficiency syndrome (AIDS), a life-threatening condition associated with excess mortality.

HIV infections initially begin in the gut, with the virus infecting the intestinal-associated lymphoid tissue (Dillon et al., 2016). Research over recent years has questioned the role of the intestinal microbiota in HIV infections, given that these infections are associated with translocation of the microbiota and its products across the damaged intestinal epithelial layer (Brenchley et al., 2006; Klase et al., 2015). The mechanisms by which HIV alters the gut microbiota and how the microbial population of the gut modulates differential responses to infection have previously been reviewed in detail (Dinh et al., 2015; Zilberman-Schapira et al., 2016).

Comparisons of the microbiota of HIV-infected individuals, those receiving antiretroviral treatment, or with varying CD4+ T cell levels found no significant difference in phage richness or diversity (Monaco et al., 2016). In contrast, other studies have observed a significant increase in the number of phage sequences in Simian immunodeficiency virus (SIV)-infected rhesus monkeys, a commonly used animal model of HIV (Handley et al., 2012). As phages have been shown to directly interact with and modulate immune cells, the contribution of phages to HIV infection should be investigated further (Foca et al., 2015).

The plasma of HIV/AIDS patients was dominated by phages and bacteria similar to those found in the human gut, whereas healthy controls showed no detectable phages in their plasma virome (Li et al., 2012). A high proportion of this HIV-associated plasma virome was composed of Enterobacteria and Pseudomonas phages (Li et al., 2012). This could suggest that phages and bacteria leak from the gut during HIV infection and move into systemic tissues.

The antiretroviral treatment for HIV could potentially have unintended effects on the phageome. An increase in gut dysbiosis has been reported upon administration of antiretroviral therapy, indicating possible consequences for gut bacteria and their phages (Klase et al., 2015). If the gut phage populations differ in HIV-infected individuals, this could have implications for their response to therapy. The ability of phages to influence the leaky gut (Li et al., 2012) must also be considered, as it has been shown that HIV patients with increased gut permeability may not respond to antiretroviral therapy (Marchetti et al., 2008).

\section{Cardiovascular Disease (CVD)}

CVD is a broad term used to define several diseases affecting the cardiovascular system, including ischaemic heart disease, myocardial infarction, and congestive cardiac failure. The driving forces behind the development of CVD are 
atherosclerosis and hypertension, which are themselves intricately related. Atherosclerosis is an inflammatory response characterised by macrophage activation and Th1 responses, among many other contributing factors. The aetiology of CVD can be bacterial, with direct links between the bacterial metabolites and risk of atherosclerotic cardiovascular disease (Wang et al., 2011).

Changes in the gut phageome are more common in patients with CVD and hypertension (Jie et al., 2017; Han et al., 2018). The presence of chronic inflammation is a driver of atherosclerosis and is seen in many autoimmune diseases (Yarur et al., 2011; Escarcega et al., 2018), and some phages may have a contributory effect. Phages can induce the production of IFN- $\gamma$, a key cytokine in macrophage activation. An increase in serum phage load was demonstrated in patients with CVD which potentially plays a role in the driving inflammatory response of the disease (Dinakaran et al., 2014). Oral administration of phage cocktails has been shown to increase gut permeability (Tetz et al., 2017). Subsequently, gut permeability can lead to increased bacteria and bacteriophages in the bloodstream, with potential synergistic effects in congestive cardiac failure (Sandek et al., 2007). To complicate this effect, different phage cocktails induce varying cytokine profile alterations. Oral administration of T7 phages increased serum cytokines such as IL-1 $\alpha$, IL-1 $\beta$ IL-2, IL12 and IL-17 (Park et al., 2014). However, the role of such cytokines in cardiovascular disease is controversial with evidence of both pro- and anti-atherosclerotic effects (Gong et al., 2015; Williams et al., 2019). Therefore, the role of phages in the development of cardiovascular disease may be due to modulatory effects on the inflammatory response.

Conversely, there is evidence that phages may have a protective role against CVD. Swinepox virus (a eukaryotic virus) has been shown to reduce the incidence of restenosis following stent insertion, a common treatment option for patients with angina pectoris and myocardial infarction (Shimamura et al., 2012). If this is true of eukaryotic viruses, there are potentially similar effects with bacteriophages also. Phage as medical tools, such as phage display, can be utilised for CVD diagnostics (Park et al., 2010) and drug delivery (Nicol et al., 2009). Phage therapy can be used to prevent or treat postheart surgery infections (Potapov et al., 2020), as well as altering the detrimental metabolism of bacteria in high-risk patients.

As CVD is complex in its aetiology, it is difficult to define the specific role phages may play in the disease. However, it seems clear that phage communities vary between healthy and CVD populations, in addition to their immunomodulatory role, suggesting this area is worthy of further investigation.

\section{Autism Spectrum Disorder (ASD)}

ASD is a developmental disorder characterised by significant variation in social, communication and behavioural traits. Gut microbiota changes have been observed in patients with ASD, typified by reduced biodiversity and changes in gut anaerobic bacteria abundance, potentially contributing to the disorder's severity (Parracho et al., 2005; Krajmalnik-Brown et al., 2015).

An alteration in gut permeability is a hypothesis for the development of several diseases, including ASD, and the effects of phages on the gut microbiome may provide a mechanism for this (Tetz and Tetz, 2016). A trail of a modified FMT treatment showed gut bacterial populations of individuals with ASD became more similar to healthy populations, whereas phage populations remained more diverse; these changes were linked with an improvement in ASD symptoms (Kang et al., 2017). The gut phageome is known to change through childhood (see section Development of the Phageome) (Figure 3), and it is possible that gut dysbiosis during childhood may contribute to the development or severity of ASD, as well as several other psychiatric disorders (Lim et al., 2016; Sherwin et al., 2016; Dickerson et al., 2017). For example, Lactobacillus phage $\varphi$ adh has been found to be more common in the oropharyngeal microbiome in patients with schizophrenia (Sherwin et al., 2016).

Psychiatric disorders are extremely complex, with the direct causes remaining unknown. From current evidence it appears that phages, as part of the larger gut microbiome community, are likely to play a role at some level, which may become clearer with future research.

\section{Does the Phageome Play a Role in Human Gut Health?}

An increase in the abundance of gut phages appears to be a common theme across the diseases reviewed here (Table 6). There is a general shift in the phageome in CD and UC to an increased number of Caudovirales, with an expansion of a few particular phage species (Lepage et al., 2008; Wagner et al., 2013; Norman et al., 2015; Fernandes et al., 2019; Zuo et al., 2019). However, studies of the phageome in other human diseases supported evidence that an increase in specific phage species correlated with disease (Li et al., 2012; Breitbart et al., 2018; Tetz et al., 2019). This could suggest that the role of phages in human disease may be specific to particular gut phage-host relationships, rather than an overall trend within the entire gut phage community.

Another common theme is a strong association with dysregulated inflammation underlying disease, which potentially could elicit phageome's influence on human disease. Phage proteins can directly induce gut inflammation (Norman et al., 2014; Majewska et al., 2015; Miernikiewicz et al., 2016), but whether this is a response to changes in the gut microbiome, or a driving factor of gut dysbiosis, is yet to be determined.

Bacterial communities of the gut are the most studied for their impact on human health and disease, recent research has further highlighted other populations in the gut communities, including fungal (Nash et al., 2017) and archaeal communities (Dridi et al., 2011). Research into the phageome, to date, has aimed to understand fluctuations in the abundance and diversity of phage groups, and how these may correlate with disease. However, we are yet to identify any specific functional relationships between phages and human disease. Moving forward, research into the mechanisms by which the gut phageome influences disease is necessary and may help answer a number of vital questions. Are specific phage species involved in causing certain health conditions, or is disease influenced by the dynamics of the phageome as a whole? What precise biological mechanisms are behind phage immunomodulation? 
Are phages the driving force behind disease, or are fluctuations in the individual phageome a result of changing gut bacterial populations? The conditions discussed in this review are complex and are often associated with individual genetics combined with environmental triggers; how does the impact of the phageome vary between individuals in the context of disease? Resolving these questions will provide a clearer view of the functional relationship between the phageome and human disease.

Research so far has presented data on how both phage and bacterial populations change in comparison to healthy controls. While this has highlighted areas in which these microbial gut communities influence disease, we are not frequently given a longitudinal perspective about how these communities change with progression from a healthy state to diseased. As earlier stated (see section Impact of Medical Interventions on the Phageome), there is limited scope for establishing what can be described as a "core phageome", compared to our knowledge of gut bacterial populations. Variation between individuals is great, and therefore elucidating if changes in phage populations are due to a single factor is difficult. Longitudinal studies are becoming more common, with metagenomic studies of UC and CD expanding, which may help to define a core phageome and establish biomarkers of disease (Norman et al., 2015; Manrique et al., 2016; Clooney et al., 2019). However the chicken-or-egg argument remains; which came first, the changes in the microbiome or did the microbiome adapt to the disease? The phageome adds another layer of complexity to this discussion, did the phageome, bacterial host or human disease initiate these changes? By using model systems such as mouse studies, we may be able to more easily manipulate these factors to unpick the true workings of the gut phageome. However, reducing gut phageome studies to these less complex systems eliminates the influence of the multifaceted human life and cannot be fully reflective of the true workings of the phageome.

Despite the microbiome receiving much more in-depth research than the phaegome, limited applications to prevent or treat diseases exist (Durack and Lynch, 2019). Therefore, it is likely to be a long time before any therapeutic options for curing systemic disease by altering the phageome become available. Perhaps understanding any perturbations of the phageome and microbiome following the application of a medical treatments, such as phage therapy, would provide insight into potential new therapies focussed on the phageome.

\section{CONCLUSIONS}

In this paper we have shown there is a wealth of literature on the role of bacteriophages within the human gut. However, there are still large areas which remain unknown or complex, which with further investigation could lead to discoveries that inform beneficial treatments for human disease, in a similar fashion as we have seen with the microbiome.

The methods used to study phages influence the communities detected. With recent advances in technology studying in-depth metagenomes, it is becoming easier to elucidate how these viral populations change and are influenced by their environment. A prominent example of these new discoveries are CrAssphage, recent work has highlighted its existence as an important component of the human gut phageome. These are just one group out of many phages in the diverse populations of microorganisms that colonize our gastrointestinal tract from birth.

We are discovering that phage populations are highly specific to individuals and the existence of a core phageome, like the microbiome, is a loose term. Phages perform a number of functions in the human gut including: genetic exchange, maintenance of diversity, supply of nutrients and interactions with the immune system. Additionally, host and virus dynamics are highly complex and vary depending on the context in which predator and prey or symbionts meet. Although an understudied area, it does appear that phage populations vary between healthy individuals and disease states. It is well understood that the micro- and mycobiomes influence a variety of human conditions; this also applies to the phageome. Further research is warranted to establish if phage manipulation would be beneficial in these conditions.

From the evidence discussed in this paper, we believe that the role and impact of the phageome is underexplored, and with continued investigation potential therapies and a deeper understanding of the viral influence on human health can be discovered.

\section{Perspectives and Future Directions}

Since the importance of the gut microbiome has come to light over the past decade, we have increased our understanding of the function of the human body and how it is not solely "human". The reliance we have on microbial communities for even our basic biology has become abundantly clear. However, as discussed throughout this paper, the largely overlooked phageome evidently plays a major role.

We hope that with the continuing improvements in the availability and accessibility to genomic research methods, the workings of the phageome will be increasingly understood as we have seen with the microbiome. As discussed in this paper, DNA technologies are often utilised, but RNA techniques are still lacking. Unfortunately this leaves a portion of the phageome without proper characterisation.

Discovering how these communities interact and interplay with their human host may lead to improvements in health, nutrition and wellbeing through manipulation of these microscopic communities. We believe this is of heightened importance in light of increasing antimicrobial resistance as traditional antibiotic therapies may become redundant. Phage therapy has already been established as a viable alternative treatment and has found a recent resurgence in popularity in Western medicine. We hope this therapy could be applied in complex diseases such as those discussed in this paper, in which the gut microbiota is disturbed. Understanding more about the phageome will in turn further our knowledge of human health, leading to a brighter future for human health. 


\section{AUTHOR CONTRIBUTIONS}

EJ conceived, designed and critically reviewed the manuscript. The majority of the manuscript was written by ET, while LK, $\mathrm{GM}, \mathrm{JB}, \mathrm{NH}$, and DL contributed specialist sections. The figures and tables were compiled by EJ, ET, LK, GM, NH, and DL. All authors contributed to the article and approved the submitted version.

\section{REFERENCES}

Abeles, S. R., Ly, M., Santiago-Rodriguez, T. M., and Pride, D. T. (2015). Effects of Long Term Antibiotic Therapy on Human Oral and Fecal Viromes. PloS One 10, e0134941. doi: 10.1371/journal.pone.0134941

Adriaenssens, E. M., and Cowan, D. A. (2014). Using Signature Genes as Tools to Assess Environmental Viral Ecology and Diversity. Appl. Environ. Microbiol. 80, 4470-4480. doi: 10.1128/AEM.00878-14

Aggarwala, V., Liang, G., and Bushman, F. D. (2017). Viral Communities of the Human Gut: Metagenomic Analysis of Composition and Dynamics. Mob DNA 8, 12. doi: 10.1186/s13100-017-0095-y

Akçelik, M. (1998). A Phage DNA Injection-Blocking Type Resistance Mechanism Encoded by Chromosomal DNA in Lactococcus Lactis Subsp. Lactis PLM-18. Milchwissenschaft 53, 619-622.

Allen, H. K., Looft, T., Bayles, D. O., Humphrey, S., Levine, U. Y., Alt, D., et al. (2011). Antibiotics in Feed Induce Prophages in Swine Fecal Microbiomes. mBio 2 (6), e00260-11. doi: 10.1128/mBio.00260-11

Allers, E., Moraru, C., Duhaime, M. B., Beneze, E., Solonenko, N., Barrero-Canosa, J., et al. (2013). Single-Cell and Population Level Viral Infection Dynamics Revealed by Phage FISH, a Method to Visualize Intracellular and Free Viruses. Environ. Microbiol. 15, 2306-2318. doi: 10.1111/1462-2920.12100

Allwood, P. B., Malik, Y. S., Maherchandani, S., Vought, K., Johnson, L. A., Braymen, C., et al. (2004). Occurrence of Escherichia Coli, Noroviruses, and F-Specific Coliphages in Fresh Market-Ready Produce. J. Food Prot. 67, 23872390. doi: $10.4315 / 0362-028 x-67.11 .2387$

American Diabetes Association. (2009). Diagnosis and Classification of Diabetes Mellitus. Diabetes Care 32 (Supplement 1), S62-S67. doi: 10.2337/dc09-S062

Andoh, A., Imaeda, H., Aomatsu, T., Inatomi, O., Bamba, S., Sasaki, M., et al. (2011). Comparison of the Fecal Microbiota Profiles Between Ulcerative Colitis and Crohn's Disease Using Terminal Restriction Fragment Length Polymorphism Analysis. J. Gastroenterol. 46, 479-486. doi: 10.1007/s00535010-0368-4

Armon, R., Araujo, R., Kott, Y., Lucena, F., and Jofre, J. (1997). Bacteriophages of Enteric Bacteria in Drinking Water, Comparison of Their Distribution in Two Countries. J. Appl. Microbiol. 83, 627-633. doi: 10.1046/j.1365-2672.1997. 00278.x

Arnold, J. W., Roach, J., and Azcarate-Peril, M. A. (2016). Emerging Technologies for Gut Microbiome Research. Trends Microbiol. 24, 887-901. doi: 10.1016/ j.tim.2016.06.008

Balcazar, J. L. (2014). Bacteriophages as Vehicles for Antibiotic Resistance Genes in the Environment. PloS Pathog. 10, e1004219. doi: 10.1371/journal.ppat. 1004219

Barr, J. J., Auro, R., Furlan, M., Whiteson, K. L., Erb, M. L., Pogliano, J., et al. (2013a). Bacteriophage Adhering to Mucus Provide a Non-Host-Derived Immunity. Proc. Natl. Acad. Sci. U. S. A. 110, 10771-10776. doi: 10.1073/ pnas. 1305923110

Barr, J. J., Auro, R., Sam-Soon, N., Kassegne, S., Peters, G., Bonilla, N., et al. (2015). Subdiffusive Motion of Bacteriophage in Mucosal Surfaces Increases the Frequency of Bacterial Encounters. Proc. Natl. Acad. Sci. U. S. A. 112, 13675-13680. doi: 10.1073/pnas.1508355112

Barr, J. J., Youle, M., and Rohwer, F. (2013b). Innate and Acquired BacteriophageMediated Immunity. Bacteriophage 3, e25857. doi: 10.4161/bact.25857

Baugher, J. L., Durmaz, E., and Klaenhammer, T. R. (2014). Spontaneously Induced Prophages in Lactobacillus Gasseri Contribute to Horizontal Gene Transfer. Appl. Environ. Microbiol. 80, 3508-3517. doi: 10.1128/AEM. 04092-13

\section{FUNDING}

This work was supported by Warwick Integrative Synthetic Biology (WISB), funded jointly by BBSRC/EPSRC, grant ref: BB/M017982/1 under the UK Research Councils' Synthetic Biology for Growth programme. The work has also been supported by $\mathrm{PhD}$ fellowships awarded to LK, GM, and JB through DTPs funded by BBSRC, EPSRC and NERC.

Bikel, S., Valdez-Lara, A., Cornejo-Granados, F., Rico, K., Canizales-Quinteros, S., Soberon, X., et al. (2015). Combining Metagenomics, Metatranscriptomics and Viromics to Explore Novel Microbial Interactions: Towards a Systems-Level Understanding of Human Microbiome. Comput. Struct. Biotechnol. J. 13, 390401. doi: $10.1016 /$ j.csbj.2015.06.001

Bojanova, D. P., and Bordenstein, S. R. (2016). Fecal Transplants: What Is Being Transferred? PloS Biol. 14, e1002503. doi: 10.1371/journal.pbio.1002503

Bonilla, N., Santiago, T., Marcos, P., Urdaneta, M., Domingo, J. S., and Toranzos, G. A. (2010). Enterophages, a Group of Phages Infecting Enterococcus Faecalis, and Their Potential as Alternate Indicators of Human Faecal Contamination. Water Sci. Technol. 61, 293-300. doi: 10.2166/wst.2010.815

Brady, A., and Salzberg, S. L. (2009). Phymm and PhymmBL: Metagenomic Phylogenetic Classification With Interpolated Markov Models. Nat. Methods 6, 673-676. doi: 10.1038/nmeth.1358

Breitbart, M., Bonnain, C., Malki, K., and Sawaya, N. A. (2018). Phage Puppet Masters of the Marine Microbial Realm. Nat. Microbiol. 3, 754-766. doi: 10.1038/s41564-018-0166-y

Breitbart, M., Haynes, M., Kelley, S., Angly, F., Edwards, R. A., Felts, B., et al. (2008). Viral Diversity and Dynamics in an Infant Gut. Res. Microbiol. 159, 367-373. doi: 10.1016/j.resmic.2008.04.006

Breitbart, M., Hewson, I., Felts, B., Mahaffy, J. M., Nulton, J., Salamon, P., et al. (2003). Metagenomic Analyses of an Uncultured Viral Community From Human Feces. J. Bacteriol. 185, 6220-6223. doi: 10.1128/jb.185.20.62206223.2003

Brenchley, J. M., Price, D. A., Schacker, T. W., Asher, T. E., Silvestri, G., Rao, S. et al. (2006). Microbial Translocation Is a Cause of Systemic Immune Activation in Chronic HIV Infection. Nat. Med. 12, 1365-1371. doi: $10.1038 / \mathrm{nm} 1511$

Brown, M. R., Camezuli, S., Davenport, R. J., Petelenz-Kurdziel, E., Ovreas, L., and Curtis, T. P. (2015). Flow Cytometric Quantification of Viruses in Activated Sludge. Water Res. 68, 414-422. doi: 10.1016/j.watres.2014.10.018

Bruttin, A., and Brussow, H. (2005). Human Volunteers Receiving Escherichia Coli Phage T4 Orally: A Safety Test of Phage Therapy. Antimicrob. Agents Chemother. 49, 2874-2878. doi: 10.1128/AAC.49.7.2874-2878.2005

Bullas, L., Colson, C., and VAN PEL, A. (1976). DNA Restriction and Modification Systems in Salmonella.* SQ, A New System Derived by Recombination Between the SB System of Salmonella Typhimurium and the SP System of Salmonella Potsdam. Microbiology 95, 166-172. doi: 10.1099/00221287-95-1-166

Callanan, J., Stockdale, S. R., Shkoporov, A., Draper, L. A., Ross, R. P., and Hill, C. (2018). RNA Phage Biology in a Metagenomic Era. Viruses 10, 386. doi: $10.3390 / \mathrm{v} 10070386$

Callanan, J., Stockdale, S., Shkoporov, A., Draper, L., Ross, R., and Hill, C. (2020). Expansion of Known ssRNA Phage Genomes: From Tens to Over a Thousand. Sci. Adv. 6, eaay5981. doi: 10.1126/sciadv.aay5981

Camacho, C., Coulouris, G., Avagyan, V., Ma, N., Papadopoulos, J., Bealer, K., et al. (2009). BLAST+: Architecture and Applications. BMC Bioinform. 10, 1-9. doi: 10.1186/1471-2105-10-421

Camarillo-Guerrero, L. F., Almeida, A., Rangel-Pineros, G., Finn, R. D., and Lawley, T. D. (2021). Massive Expansion of Human Gut Bacteriophage Diversity. Cell 184, 1098-1109. doi: 10.1016/j.cell.2021.01.029. e1099.

Carrolo, M., Frias, M. J., Pinto, F. R., Melo-Cristino, J., and Ramirez, M. (2010). Prophage Spontaneous Activation Promotes DNA Release Enhancing Biofilm Formation in Streptococcus Pneumoniae. PloS One 5, e15678. doi: 10.1371/ journal.pone. 0015678

Castro-Mejia, J. L., Muhammed, M. K., Kot, W., Neve, H., Franz, C. M., Hansen, L. H., et al. (2015). Optimizing Protocols for Extraction of Bacteriophages Prior to 
Metagenomic Analyses of Phage Communities in the Human Gut. Microbiome 3, 64. doi: 10.1186/s40168-015-0131-4

Cheng, G., Hao, H., Xie, S., Wang, X., Dai, M., Huang, L., et al. (2014). Antibiotic Alternatives: The Substitution of Antibiotics in Animal Husbandry? Front. Microbiol. 5, 217. doi: 10.3389/fmicb.2014.00217

Chopin, M.-C., Chopin, A., and Bidnenko, E. (2005). Phage Abortive Infection in Lactococci: Variations on a Theme. Curr. Opin. Microbiol. 8, 473-479. doi: 10.1016/j.mib.2005.06.006

Chukeatirote, E., Phongtang, W., Kim, J., Jo, A., Jung, L. S., and Ahn, J. (2018). Significance of Bacteriophages in Fermented Soybeans: A Review. Biomol Concepts 9, 131-142. doi: 10.1515/bmc-2018-0012

Cinek, O., Kramna, L., Lin, J., Oikarinen, S., Kolarova, K., Ilonen, J., et al. (2017). Imbalance of Bacteriome Profiles Within the Finnish Diabetes Prediction and Prevention Study: Parallel Use of 16 S Profiling and Virome Sequencing in Stool Samples From Children With Islet Autoimmunity and Matched Controls. Pediatr. Diabetes 18, 588-598. doi: 10.1111/pedi. 12468

Clooney, A. G., Sutton, T. D., Shkoporov, A. N., Holohan, R. K., Daly, K. M., O'Regan, O., et al. (2019). Whole-Virome Analysis Sheds Light on Viral Dark Matter in Inflammatory Bowel Disease. Cell Host Microbe 26, 764-778. doi: 10.1016/j.chom.2019.10.009. e765.

Colson, P., Richet, H., Desnues, C., Balique, F., Moal, V., Grob, J. J., et al. (2010). Pepper Mild Mottle Virus, A Plant Virus Associated With Specific Immune Responses, Fever, Abdominal Pains, and Pruritus in Humans. PloS One 5, e10041. doi: 10.1371/journal.pone.0010041

Conceicao-Neto, N., Zeller, M., Lefrere, H., De Bruyn, P., Beller, L., Deboutte, W., et al. (2015). Modular Approach to Customise Sample Preparation Procedures for Viral Metagenomics: A Reproducible Protocol for Virome Analysis. Sci. Rep. 5, 16532. doi: 10.1038/srep16532

Cornuault, J. K., Petit, M. A., Mariadassou, M., Benevides, L., Moncaut, E., Langella, P., et al. (2018). Phages Infecting Faecalibacterium Prausnitzii Belong to Novel Viral Genera That Help to Decipher Intestinal Viromes. Microbiome 6, 65. doi: 10.1186/s40168-018-0452-1

Costerton, J. W., Cheng, K. J., Geesey, G. G., Ladd, T. I., Nickel, J. C., Dasgupta, M., et al. (1987). Bacterial Biofilms in Nature and Disease. Annu. Rev. Microbiol. 41, 435-464. doi: 10.1146/annurev.mi.41.100187.002251

Dang, V. T., and Sullivan, M. B. (2014). Emerging Methods to Study Bacteriophage Infection at the Single-Cell Level. Front. Microbiol. 5, 724. doi: 10.3389/ fmicb.2014.00724

David, L. A., Maurice, C. F., Carmody, R. N., Gootenberg, D. B., Button, J. E., Wolfe, B. E., et al. (2014). Diet Rapidly and Reproducibly Alters the Human Gut Microbiome. Nature 505, 559-563. doi: 10.1038/nature12820

Davies, E. V., James, C. E., Williams, D., O’Brien, S., Fothergill, J. L., Haldenby, S., et al. (2016). Temperate Phages Both Mediate and Drive Adaptive Evolution in Pathogen Biofilms. Proc. Natl. Acad. Sci. U. S. A. 113, 8266-8271. doi: 10.1073/ pnas. 1520056113

Debroas, D., and Siguret, C. (2019). Viruses as Key Reservoirs of Antibiotic Resistance Genes in the Environment. ISME J. 13, 2856-2867. doi: 10.1038/ s41396-019-0478-9

Degnan, P. H., Barry, N. A., Mok, K. C., Taga, M. E., and Goodman, A. L. (2014). Human Gut Microbes Use Multiple Transporters to Distinguish Vitamin B12 Analogs and Compete in the Gut. Cell Host Microbe 15, 47-57. doi: 10.1016/ j.chom.2013.12.007

Denou, E., Pridmore, R. D., Ventura, M., Pittet, A.-C., Zwahlen, M.-C., Berger, B., et al. (2008). The Role of Prophage for Genome Diversification Within a Clonal Lineage of Lactobacillus Johnsonii: Characterization of the Defective Prophage Lj771. J. Bacteriol. 190, 5806. doi: 10.1128/ JB.01802-07

Deriu, E., Liu, J. Z., Pezeshki, M., Edwards, R. A., Ochoa, R. J., Contreras, H., et al. (2013). Probiotic Bacteria Reduce Salmonella Typhimurium Intestinal Colonization by Competing for Iron. Cell Host Microbe 14, 26-37. doi: 10.1016/j.chom.2013.06.007

De Sordi, L., Lourenço, M., and Debarbieux, L. (2019). "I Will Survive": A Tale of Bacteriophage-Bacteria Coevolution in the Gut. Gut Microbes 10, 92-99. doi: 10.1080/19490976.2018.1474322

Destoumieux-Garzón, D., Duquesne, S., Peduzzi, J., Goulard, C., Desmadril, M., Letellier, L., et al. (2005). The Iron-Siderophore Transporter FhuA Is the Receptor for the Antimicrobial Peptide Microcin J25: Role of the Microcin Val11-Pro16 $\beta$-Hairpin Region in the Recognition Mechanism. Biochem. J. 389, 869-876. doi: 10.1042/BJ20042107

Deveau, H., Barrangou, R., Garneau, J. E., Labonté, J., Fremaux, C., Boyaval, P., et al. (2008). Phage Response to CRISPR-Encoded Resistance in Streptococcus Thermophilus. J. Bacteriol. 190, 1390-1400. doi: 10.1128/JB.01412-07

Devoto, A. E., Santini, J. M., Olm, M. R., Anantharaman, K., Munk, P., Tung, J., et al. (2019). Megaphages Infect Prevotella and Variants Are Widespread in Gut Microbiomes. Nat. Microbiol. 4, 693-700. doi: 10.1038/s41564-018-0338-9

d'Herelle, F. (1918). Technique De La Recherche Du Microbe Filtrant Bactériophage (Bacteriophagum Intestinale). CR Soc Biol. 81, 1160-1162.

d'Herelle, F. (1919). Sur Le Microbe Bactériophage. Comptes Rendus La Société Biol. 82, 1237.

d'Hérelle, F. (1921). Le bactériophage; son rôle dans l'immunité (Paris, France: Masson et cie).

Diard, M., Bakkeren, E., Cornuault, J. K., Moor, K., Hausmann, A., Sellin, M. E., et al. (2017). Inflammation Boosts Bacteriophage Transfer Between Salmonella Spp. Science 355, 1211-1215. doi: 10.1126/science.aaf8451

Dickerson, F., Severance, E., and Yolken, R. (2017). The Microbiome, Immunity, and Schizophrenia and Bipolar Disorder. Brain Behav. Immun. 62, 46-52. doi: 10.1016/j.bbi.2016.12.010

Dillon, S. M., Frank, D. N., and Wilson, C. C. (2016). The Gut Microbiome and HIV-1 Pathogenesis: A Two-Way Street. AIDS 30, 2737-2751. doi: 10.1097/ QAD.0000000000001289

Dinakaran, V., Rathinavel, A., Pushpanathan, M., Sivakumar, R., Gunasekaran, P., and Rajendhran, J. (2014). Elevated Levels of Circulating DNA in Cardiovascular Disease Patients: Metagenomic Profiling of Microbiome in the Circulation. PloS One 9, e105221. doi: 10.1371/journal.pone.0105221

Dinh, D. M., Volpe, G. E., Duffalo, C., Bhalchandra, S., Tai, A. K., Kane, A. V., et al. (2015). Intestinal Microbiota, Microbial Translocation, and Systemic Inflammation in Chronic HIV Infection. J. Infect. Dis. 211, 19-27. doi: 10.1093/infdis/jiu409

Donaldson, G. P., Lee, S. M., and Mazmanian, S. K. (2016). Gut Biogeography of the Bacterial Microbiota. Nat. Rev. Microbiol. 14, 20-32. doi: 10.1038/nrmicro3552

Douglas, A. E. (2019). Simple Animal Models for Microbiome Research. Nat. Rev. Microbiol. 17, 764-775. doi: 10.1038/s41579-019-0242-1

Doulatov, S., Hodes, A., Dai, L., Mandhana, N., Liu, M., Deora, R., et al. (2004). Tropism Switching in Bordetella Bacteriophage Defines a Family of DiversityGenerating Retroelements. Nature 431, 476-481. doi: 10.1038/nature02833

Draper, L. A., Ryan, F., Dalmasso, M., Casey, P. G., Mccann, A., Vimalkumar, V., et al. (2020). Autochthonous Faecal Virome Transplantation (FVT) Reshapes the Murine Microbiome After Antibiotic Perturbation. BMC Biol. 18, 173. doi: 10.1186/s12915-020-00906-0

Dridi, B., Raoult, D., and Drancourt, M. (2011). Archaea as Emerging Organisms in Complex Human Microbiomes. Anaerobe 17, 56-63. doi: 10.1016/ j.anaerobe.2011.03.001

Duerkop, B. A., Clements, C. V., Rollins, D., Rodrigues, J. L., and Hooper, L. V. (2012). A Composite Bacteriophage Alters Colonization by an Intestinal Commensal Bacterium. Proc. Natl. Acad. Sci. U. S. A. 109, 17621-17626. doi: 10.1073/pnas. 1206136109

Duerkop, B. A., Kleiner, M., Paez-Espino, D., Zhu, W., Bushnell, B., Hassell, B., et al. (2018). Murine Colitis Reveals a Disease-Associated Bacteriophage Community. Nat. Microbiol. 3, 1023-1031. doi: 10.1038/s41564-018-0210-y

Duffy, S., Shackelton, L. A., and Holmes, E. C. (2008). Rates of Evolutionary Change in Viruses: Patterns and Determinants. Nat. Rev. Genet. 9, 267-276. doi: $10.1038 / \mathrm{nrg} 2323$

Durack, J., and Lynch, S. V. (2019). The Gut Microbiome: Relationships With Disease and Opportunities for Therapy. J. Exp. Med. 216, 20-40. doi: 10.1084/ jem. 20180448

Duranti, S., Lugli, G. A., Mancabelli, L., Armanini, F., Turroni, F., James, K., et al. (2017). Maternal Inheritance of Bifidobacterial Communities and Bifidophages in Infants Through Vertical Transmission. Microbiome 5, 66. doi: 10.1186/ s40168-017-0282-6

Dutilh, B. E., Cassman, N., McNair, K., Sanchez, S. E., Silva, G. G., Boling, L., et al. (2014). A Highly Abundant Bacteriophage Discovered in the Unknown Sequences of Human Faecal Metagenomes. Nat. Commun. 5, 4498. doi: $10.1038 /$ ncomms 5498

Džunková, M., Low, S. J., Daly, J. N., Deng, L., Rinke, C., and Hugenholtz, P. (2019). Defining the Human Gut Host-Phage Network Through Single-Cell Viral Tagging. Nat. Microbiol. 4, 2192-2203. doi: 10.1038/s41564-019-0526-2 
Eckburg, P. B., Bik, E. M., Bernstein, C. N., Purdom, E., Dethlefsen, L., Sargent, M., et al. (2005). Diversity of the Human Intestinal Microbial Flora. Science 308, 1635-1638. doi: 10.1126/science.1110591

Eddy, S. R. (2011). Accelerated Profile HMM Searches. PloS Comput. Biol. 7, e1002195. doi: 10.1371/journal.pcbi.1002195

Edwards, R. A., Vega, A. A., Norman, H. M., Ohaeri, M., Levi, K., Dinsdale, E. A., et al. (2019). Global Phylogeography and Ancient Evolution of the Widespread Human Gut Virus Crassphage. Nat. Microbiol. 4, 1727-1736. doi: 10.1038/ s41564-019-0494-6

El-Gebali, S., Mistry, J., Bateman, A., Eddy, S. R., Luciani, A., Potter, S. C., et al. (2019). The Pfam Protein Families Database in 2019. Nucleic Acids Res. 47, D427-D432. doi: 10.1093/nar/gky995

Enault, F., Briet, A., Bouteille, L., Roux, S., Sullivan, M. B., and Petit, M.-A. (2017). Phages Rarely Encode Antibiotic Resistance Genes: A Cautionary Tale for Virome Analyses. ISME J. 11, 237-247. doi: 10.1038/ismej.2016.90

Escarcega, R. O., Lipinski, M. J., Garcia-Carrasco, M., Mendoza-Pinto, C., GalvezRomero, J. L., and Cervera, R. (2018). Inflammation and Atherosclerosis: Cardiovascular Evaluation in Patients With Autoimmune Diseases. Autoimmun. Rev. 17, 703-708. doi: 10.1016/j.autrev.2018.01.021

Fernandes, M. A., Verstraete, S. G., Phan, T. G., Deng, X., Stekol, E., LaMere, B., et al. (2019). Enteric Virome and Bacterial Microbiota in Children With Ulcerative Colitis and Crohn Disease. J. Pediatr. Gastroenterol. Nutr. 68, 3036. doi: 10.1097/MPG.0000000000002140

Fineran, P. C., Blower, T. R., Foulds, I. J., Humphreys, D. P., Lilley, K. S., and Salmond, G. P. (2009). The Phage Abortive Infection System, ToxIN, Functions as a Protein-RNA Toxin-Antitoxin Pair. Proc. Natl. Acad. Sci. U. S. A. 106, 894-899. doi: 10.1073/pnas.0808832106

Flemming, H.-C., and Wingender, J. (2010). The Biofilm Matrix. Nat. Rev. Microbiol. 8, 623-633. doi: 10.1038/nrmicro2415

Flewett, T. H., Bryden, A. S., and Davies, H. (1974). Diagnostic Electron Microscopy of Faeces. I. The Viral Flora of the Faeces as Seen by Electron Microscopy. J. Clin. Pathol. 27, 603-608. doi: 10.1136/jcp.27.8.603

Foca, A., Liberto, M. C., Quirino, A., Marascio, N., Zicca, E., and Pavia, G. (2015). Gut Inflammation and Immunity: What Is the Role of the Human Gut Virome? Mediators Inflamm. 2015:326032. doi: 10.1155/2015/326032

Forterre, P., Soler, N., Krupovic, M., Marguet, E., and Ackermann, H.-W. (2013). Fake Virus Particles Generated by Fluorescence Microscopy. Trends Microbiol. 21, 1-5. doi: 10.1016/j.tim.2012.10.005

Fraser, J. S., Maxwell, K. L., and Davidson, A. R. (2007). Immunoglobulin-Like Domains on Bacteriophage: Weapons of Modest Damage? Curr. Opin. Microbiol. 10, 382-387. doi: 10.1016/j.mib.2007.05.018

Furuse, K., Osawa, S., Kawashiro, J., Tanaka, R., Ozawa, A., Sawamura, S., et al. (1983). Bacteriophage Distribution in Human Faeces: Continuous Survey of Healthy Subjects and Patients With Internal and Leukaemic Diseases. J. Gen. Virol. 64 (Pt 9), 2039-2043. doi: 10.1099/0022-1317-64-9-2039

Galiez, C., Siebert, M., Enault, F., Vincent, J., and Söding, J. (2017). WisH: Who Is the Host? Predicting Prokaryotic Hosts From Metagenomic Phage Contigs. Bioinformatics 33, 3113-3114. doi: 10.1093/bioinformatics/ btx383

Gantzer, C., Henny, J., and Schwartzbrod, L. (2002). Bacteroides Fragilis and Escherichia Coli Bacteriophages in Human Faeces. Int. J. Hyg Environ. Health 205, 325-328. doi: 10.1078/1438-4639-00152

Gigante, A., and Atterbury, R. J. (2019). Veterinary Use of Bacteriophage Therapy in Intensively-Reared Livestock. Virol. J. 16, 1-9. doi: 10.1186/s12985-0191260-3

Gillevet, P., Sikaroodi, M., Keshavarzian, A., and Mutlu, E. A. (2010). Quantitative Assessment of the Human Gut Microbiome Using Multitag Pyrosequencing. Chem. Biodivers. 7, 1065-1075. doi: 10.1002/cbdv.200900322

Gogokhia, L., Buhrke, K., Bell, R., Hoffman, B., Brown, D. G., Hanke-Gogokhia, C., et al. (2019). Expansion of Bacteriophages Is Linked to Aggravated Intestinal Inflammation and Colitis. Cell Host Microbe 25, 285-299. doi: 10.1016/ j.chom.2019.01.008

Goldfarb, T., Sberro, H., Weinstock, E., Cohen, O., Doron, S., Charpak-Amikam, Y., et al. (2015). BREX Is a Novel Phage Resistance System Widespread in Microbial Genomes. EMBO J. 34, 169-183. doi: 10.15252/embj.201489455

Gong, F., Liu, Z., Liu, J., Zhou, P., Liu, Y., and Lu, X. (2015). The Paradoxical Role of IL-17 in Atherosclerosis. Cell Immunol. 297, 33-39. doi: 10.1016/ j.cellimm.2015.05.007
Gorski, A., Wazna, E., Dabrowska, B. W., Dabrowska, K., Switala-Jelen, K., and Miedzybrodzki, R. (2006). Bacteriophage Translocation. FEMS Immunol. Med. Microbiol. 46, 313-319. doi: 10.1111/j.1574-695X.2006.00044.x

Grazziotin, A. L., Koonin, E. V., and Kristensen, D. M. (2016). Prokaryotic Virus Orthologous Groups (pVOGs): A Resource for Comparative Genomics and Protein Family Annotation. Nucleic Acids Res. 45, D491-D498. doi: 10.1093/ nar/gkw975

Gregory, A. C., Zablocki, O., Zayed, A. A., Howell, A., Bolduc, B., and Sullivan, M. B. (2020). The Gut Virome Database Reveals Age-Dependent Patterns of Virome Diversity in the Human Gut. Cell Host Microbe 28, 724-740.e728. doi: 10.1016/ j.chom.2020.08.003

Groth, A. C., Olivares, E. C., Thyagarajan, B., and Calos, M. P. (2000). A Phage Integrase Directs Efficient Site-Specific Integration in Human Cells. Proc. Natl. Acad. Sci. U. S. A. 97, 5995-6000. doi: 10.1073/pnas.090527097

Guerin, E., Shkoporov, A., Stockdale, S. R., Clooney, A. G., Ryan, F. J., Sutton, T. D. S., et al. (2018). Biology and Taxonomy of Crass-Like Bacteriophages, the Most Abundant Virus in the Human Gut. Cell Host Microbe 24, 653-664.e656. doi: 10.1016/j.chom.2018.10.002

Guo, J., Bolduc, B., Zayed, A. A., Varsani, A., Dominguez-Huerta, G., Delmont, T. O., et al. (2021). VirSorter2: A Multi-Classifier, Expert-Guided Approach to Detect Diverse DNA and RNA Viruses. Microbiome 9, 1-13. doi: 10.1186/ s40168-020-00990-y

Guo, M., Liu, G., Chen, J., Ma, J., Lin, J., Fu, Y., et al. (2020). Dynamics of Bacteriophages in Gut of Giant Pandas Reveal a Potential Regulation of Dietary Intake on Bacteriophage Composition. Sci. Total Environ. 734, 139424. doi: 10.1016/j.scitotenv.2020.139424

Handley, S. A., Thackray, L. B., Zhao, G., Presti, R., Miller, A. D., Droit, L., et al. (2012). Pathogenic Simian Immunodeficiency Virus Infection Is Associated With Expansion of the Enteric Virome. Cell 151, 253-266. doi: 10.1016/ j.cell.2012.09.024

Han, M., Yang, P., Zhong, C., and Ning, K. (2018). The Human Gut Virome in Hypertension. Front. Microbiol. 9, 3150. doi: 10.3389/fmicb.2018.03150

Hayes, S., Mahony, J., Nauta, A., and van Sinderen, D. (2017). Metagenomic Approaches to Assess Bacteriophages in Various Environmental Niches. Viruses 9:127. doi: 10.3390/v9060127

Hofer, B., Ruge, M., and Dreiseikelmann, B. (1995). The Superinfection Exclusion Gene (sieA) of Bacteriophage P22: Identification and Overexpression of the Gene and Localization of the Gene Product. J. Bacteriol. 177, 3080-3086. doi: 10.1128/jb.177.11.3080-3086.1995

Honap, T. P., Sankaranarayanan, K., Schnorr, S. L., Ozga, A. T., Warinner, C., and Lewis, C. M. Jr. (2020). Biogeographic Study of Human Gut-Associated crAssphage Suggests Impacts From Industrialization and Recent Expansion. PloS One 15, e0226930. doi: 10.1371/journal.pone.0226930

Howard, L., and Tipper, D. J. (1973). A Polypeptide Bacteriophage Receptor: Modified Cell Wall Protein Subunits in Bacteriophage-Resistant Mutants of Bacillus Sphaericus Strain P-1. J. Bacteriol. 113, 1491-1504. doi: 10.1128/ JB.113.3.1491-1504.1973

Howe, A., Ringus, D. L., Williams, R. J., Choo, Z. N., Greenwald, S. M., Owens, S. M., et al. (2016). Divergent Responses of Viral and Bacterial Communities in the Gut Microbiome to Dietary Disturbances in Mice. ISME J. 10, 1217-1227. doi: 10.1038/ismej.2015.183

Hoyles, L., McCartney, A. L., Neve, H., Gibson, G. R., Sanderson, J. D., Heller, K. J., et al. (2014). Characterization of Virus-Like Particles Associated With the Human Faecal and Caecal Microbiota. Res. Microbiol. 165, 803-812. doi: 10.1016/j.resmic.2014.10.006

Hsu, B. B., Gibson, T. E., Yeliseyev, V., Liu, Q., Lyon, L., Bry, L., et al. (2019). Dynamic Modulation of the Gut Microbiota and Metabolome by Bacteriophages in a Mouse Model. Cell Host Microbe 25, 803-814.e805. doi: 10.1016/j.chom.2019.05.001

Hughes, K. A., Sutherland, I. W., Clark, J., and Jones, M. V. (1998). Bacteriophage and Associated Polysaccharide Depolymerases-Novel Tools for Study of Bacterial Biofilms. J. Appl. Microbiol. 85, 583-590. doi: 10.1046/j.13652672.1998.853541.x

Hussain, A., Ansari, A., and Ahmad, R. (2020). "Microbial Biofilms: Human Mucosa and Intestinal Microbiota". In: New and Future Developments in Microbial Biotechnology and Bioengineering: Microbial Biofilms (Amsterdam, Netherlands: Elsevier), 47-60. doi: 10.1016/B978-0-444-64279$0.00004-9$ 
Hyatt, D., Chen, G.-L., LoCascio, P. F., Land, M. L., Larimer, F. W., and Hauser, L. J. (2010). Prodigal: Prokaryotic Gene Recognition and Translation Initiation Site Identification. BMC Bioinform. 11, 1-11. doi: 10.1186/1471-2105-11-119

Iacob, S., Iacob, D. G., and Luminos, L. M. (2019). Intestinal Microbiota as a Host Defense Mechanism to Infectious Threats. Front. Microbiol. 9, 3328. doi: $10.3389 /$ fmicb. 2018.03328

Jernberg, C., Lofmark, S., Edlund, C., and Jansson, J. K. (2010). Long-Term Impacts of Antibiotic Exposure on the Human Intestinal Microbiota. Microbiology 156, 3216-3223. doi: 10.1099/mic.0.040618-0

Jie, Z., Xia, H., Zhong, S. L., Feng, Q., Li, S., Liang, S., et al. (2017). The Gut Microbiome in Atherosclerotic Cardiovascular Disease. Nat. Commun. 8, 845. doi: 10.1038/s41467-017-00900-1

Jofre, J., Olle, E., Ribas, F., Vidal, A., and Lucena, F. (1995). Potential Usefulness of Bacteriophages That Infect Bacteroides Fragilis as Model Organisms for Monitoring Virus Removal in Drinking Water Treatment Plants. Appl. Environ. Microbiol. 61, 3227-3231. doi: 10.1128/AEM.61.9.3227-3231.1995

Jovel, J., Patterson, J., Wang, W., Hotte, N., O’Keefe, S., Mitchel, T., et al. (2016). Characterization of the Gut Microbiome Using 16S or Shotgun Metagenomics. Front. Microbiol. 7, 459. doi: 10.3389/fmicb.2016.00459

Kang, D. W., Adams, J. B., Gregory, A. C., Borody, T., Chittick, L., Fasano, A., et al. (2017). Microbiota Transfer Therapy Alters Gut Ecosystem and Improves Gastrointestinal and Autism Symptoms: An Open-Label Study. Microbiome 5:10. doi: 10.1186/s40168-016-0225-7

Kang, S., Denman, S. E., Morrison, M., Yu, Z., Dore, J., Leclerc, M., et al. (2010). Dysbiosis of Fecal Microbiota in Crohn's Disease Patients as Revealed by a Custom Phylogenetic Microarray. Inflammation Bowel Dis. 16, 2034-2042. doi: 10.1002/ibd.21319

Katoh, K., and Standley, D. M. (2013). MAFFT Multiple Sequence Alignment Software Version 7: Improvements in Performance and Usability. Mol. Biol. Evol. 30, 772-780. doi: 10.1093/molbev/mst010

Kauffman, K. M., Hussain, F. A., Yang, J., Arevalo, P., Brown, J. M., Chang, W. K., et al. (2018). A Major Lineage of Non-Tailed dsDNA Viruses as Unrecognized Killers of Marine Bacteria. Nature 554, 118-122. doi: 10.1038/nature25474

Kelley, L. A., Mezulis, S., Yates, C. M., Wass, M. N., and Sternberg, M. J. (2015). The Phyre2 Web Portal for Protein Modeling, Prediction and Analysis. Nat. Protoc. 10, 845-858. doi: 10.1038/nprot.2015.053

Kennedy, J. E.Jr., Oblinger, J. L., and Bitton, G. (1984). Recovery of Coliphages From Chicken, Pork Sausage and Delicatessen Meats. J. Food Prot. 47, 623626. doi: 10.4315/0362-028X-47.8.623

Kennedy, J.Jr., Wei, C., and Oblinger, J. (1986). Distribution of Coliphages in Various Foods. J. Food Prot. 49, 944-951. doi: 10.4315/0362-028X-49.12.944

Khor, B., Gardet, A., and Xavier, R. J. (2011). Genetics and Pathogenesis of Inflammatory Bowel Disease. Nature 474, 307-317. doi: 10.1038/nature10209

Kim, M. S., and Bae, J. W. (2018). Lysogeny Is Prevalent and Widely Distributed in the Murine Gut Microbiota. ISME J. 12, 1127-1141. doi: 10.1038/s41396-018$0061-9$

Kim, M. S., Park, E. J., Roh, S. W., and Bae, J. W. (2011). Diversity and Abundance of Single-Stranded DNA Viruses in Human Feces. Appl. Environ. Microbiol. 77, 8062-8070. doi: 10.1128/AEM.06331-11

Klase, Z., Ortiz, A., Deleage, C., Mudd, J. C., Quinones, M., Schwartzman, E., et al. (2015). Dysbiotic Bacteria Translocate in Progressive SIV Infection. Mucosal Immunol. 8, 1009-1020. doi: 10.1038/mi.2014.128

Kleiner, M., Hooper, L. V., and Duerkop, B. A. (2015). Evaluation of Methods to Purify Virus-Like Particles for Metagenomic Sequencing of Intestinal Viromes. BMC Genom. 16, 7. doi: 10.1186/s12864-014-1207-4

Kleppen, H. P., Holo, H., Jeon, S. R., Nes, I. F., and Yoon, S. S. (2012). Novel Podoviridae Family Bacteriophage Infecting Weissella Cibaria Isolated From Kimchi. Appl. Environ. Microbiol. 78, 7299-7308. doi: 10.1128/AEM.00031-12

Kortright, K. E., Chan, B. K., Koff, J. L., and Turner, P. E. (2019). Phage Therapy: A Renewed Approach to Combat Antibiotic-Resistant Bacteria. Cell Host Microbe 25, 219-232. doi: 10.1016/j.chom.2019.01.014

Koskella, B., and Brockhurst, M. A. (2014). Bacteria-Phage Coevolution as a Driver of Ecological and Evolutionary Processes in Microbial Communities. FEMS Microbiol. Rev. 38, 916-931. doi: 10.1111/1574-6976.12072

Krajmalnik-Brown, R., Lozupone, C., Kang, D. W., and Adams, J. B. (2015). Gut Bacteria in Children With Autism Spectrum Disorders: Challenges and Promise of Studying How a Complex Community Influences a Complex Disease. Microb. Ecol. Health Dis. 26, 26914. doi: 10.3402/mehd.v26.26914
Lagier, J.-C., Armougom, F., Million, M., Hugon, P., Pagnier, I., Robert, C., et al. (2012). Microbial Culturomics: Paradigm Shift in the Human Gut Microbiome Study. Clin. Microbiol. Infect. 18, 1185-1193. doi: 10.1111/1469-0691.12023

Lagier, J.-C., Hugon, P., Khelaifia, S., Fournier, P.-E., La Scola, B., and Raoult, D. (2015). The Rebirth of Culture in Microbiology Through the Example of Culturomics to Study Human Gut Microbiota. Clin. Microbiol. Rev. 28, $237-$ 264. doi: 10.1128/CMR.00014-14

Lagier, J.-C., Khelaifia, S., Alou, M. T., Ndongo, S., Dione, N., Hugon, P., et al. (2016). Culture of Previously Uncultured Members of the Human Gut Microbiota by Culturomics. Nat. Microbiol. 1, 1-8. doi: 10.1038/ nmicrobiol.2016.203

Leonard, M. T., Davis-Richardson, A. G., Ardissone, A. N., Kemppainen, K. M., Drew, J. C., Ilonen, J., et al. (2014). The Methylome of the Gut Microbiome: Disparate Dam Methylation Patterns in Intestinal Bacteroides Dorei. Front. Microbiol. 5, 361. doi: 10.3389/fmicb.2014.00361

Lepage, P., Colombet, J., Marteau, P., Sime-Ngando, T., Dore, J., and Leclerc, M. (2008). Dysbiosis in Inflammatory Bowel Disease: A Role for Bacteriophages? Gut 57, 424-425. doi: 10.1136/gut.2007.134668

Lepage, P., Seksik, P., Sutren, M., de la Cochetiere, M. F., Jian, R., Marteau, P., et al. (2005). Biodiversity of the Mucosa-Associated Microbiota Is Stable Along the Distal Digestive Tract in Healthy Individuals and Patients With IBD. Inflammation Bowel Dis. 11, 473-480. doi: 10.1097/01.mib.0000159662.62651.06

Ley, R. E., Peterson, D. A., and Gordon, J. I. (2006). Ecological and Evolutionary Forces Shaping Microbial Diversity in the Human Intestine. Cell 124, 837-848. doi: 10.1016/j.cell.2006.02.017

Liang, G., Zhao, C., Zhang, H., Mattei, L., Sherrill-Mix, S., Bittinger, K., et al. (2020). The Stepwise Assembly of the Neonatal Virome Is Modulated by Breastfeeding. Nature 581, 470-474. doi: 10.1038/s41586-020-2192-1

Li, S. K., Leung, R. K., Guo, H. X., Wei, J. F., Wang, J. H., Kwong, K. T., et al. (2012). Detection and Identification of Plasma Bacterial and Viral Elements in HIV/AIDS Patients in Comparison to Healthy Adults. Clin. Microbiol. Infect. 18, 1126-1133. doi: 10.1111/j.1469-0691.2011.03690.x

Li, H., Li, H., Wang, J., Guo, L., Fan, H., Zheng, H., et al. (2019). The Altered Gut Virome Community in Rhesus Monkeys Is Correlated With the Gut Bacterial Microbiome and Associated Metabolites. Virol. J. 16, 105. doi: 10.1186/s12985019-1211-z

Li, J., Jia, H., Cai, X., Zhong, H., Feng, Q., Sunagawa, S., et al. (2014) An Integrated Catalog of Reference Genes in the Human Gut Microbiome. Nat. Biotechnol. 32, 834-841. doi: 10.1038/nbt.2942

Lima-Mendez, G., Toussaint, A., and Leplae, R. (2011). A Modular View of the Bacteriophage Genomic Space: Identification of Host and Lifestyle Marker Modules. Res. Microbiol. 162, 737-746. doi: 10.1016/j.resmic.2011.06.006

Lim, Y. W., Schmieder, R., Haynes, M., Willner, D., Furlan, M., Youle, M., et al. (2013). Metagenomics and Metatranscriptomics: Windows on CF-Associated Viral and Microbial Communities. J. Cyst Fibros. 12, 154-164. doi: 10.1016/ j.jcf.2012.07.009

Lim, E. S., Wang, D., and Holtz, L. R. (2016). The Bacterial Microbiome and Virome Milestones of Infant Development. Trends Microbiol. 24, 801-810. doi: 10.1016/j.tim.2016.06.001

Lim, E. S., Zhou, Y., Zhao, G., Bauer, I. K., Droit, L., Ndao, I. M., et al. (2015). Early Life Dynamics of the Human Gut Virome and Bacterial Microbiome in Infants. Nat. Med. 21, 1228. doi: 10.1038/nm.3950

Lin, D. M., and Lin, H. C. (2019). A Theoretical Model of Temperate Phages as Mediators of Gut Microbiome Dysbiosis. F1000Research 8, F1000 Faculty Rev997. doi: 10.12688/f1000research.18480.1

Liu, R., Vaishnav, R. A., Roberts, A. M., and Friedland, R. P. (2013). Humans Have Antibodies Against a Plant Virus: Evidence From Tobacco Mosaic Virus. PloS One 8, e60621. doi: 10.1371/journal.pone.0060621

Looft, T., Allen, H. K., Cantarel, B. L., Levine, U. Y., Bayles, D. O., Alt, D. P., et al. (2014). Bacteria, Phages and Pigs: The Effects of In-Feed Antibiotics on the Microbiome at Different Gut Locations. ISME J. 8, 1566-1576. doi: 10.1038/ ismej.2014.12

Lozupone, C. A., Stombaugh, J. I., Gordon, J. I., Jansson, J. K., and Knight, R. (2012). Diversity, Stability and Resilience of the Human Gut Microbiota. Nature 489, 220-230. doi: 10.1038/nature11550

Lu, Z., Breidt, F., Plengvidhya, V., and Fleming, H. P. (2003). Bacteriophage Ecology in Commercial Sauerkraut Fermentations. Appl. Environ. Microbiol. 69, 3192-3202. doi: 10.1128/aem.69.6.3192-3202.2003 
Lucey, J. A. (2015). Raw Milk Consumption: Risks and Benefits. Nutr. Today 50, 189-193. doi: 10.1097/NT.0000000000000108

Lu, M.-J., and Henning, U. (1994). Superinfection Exclusion by T-even-type Coliphages. Trends Microbiol. 2, 137-139. doi: 10.1016/0966-842X(94)90601-7

Macfarlane, S., and Dillon, J. F. (2007). Microbial Biofilms in the Human Gastrointestinal Tract. J. Appl. Microbiol. 102, 1187-1196. doi: 10.1111/ j.1365-2672.2007.03287.x

Ma, C., Chen, Z., Gong, G., Huang, L., Li, S., and Ma, A. (2015). Starter Culture Design to Overcome Phage Infection During Yogurt Fermentation. Food Sci. Biotechnol. 24, 521-527. doi: 10.1007/s10068-015-0068-1

Maiques, E., Ubeda, C., Campoy, S., Salvador, N., Lasa, I., Novick, R. P., et al. (2006). Beta-Lactam Antibiotics Induce the SOS Response and Horizontal Transfer of Virulence Factors in Staphylococcus Aureus. J. Bacteriol. 188, 2726-2729. doi: 10.1128/JB.188.7.2726-2729.2006

Mai, V., Ukhanova, M., Reinhard, M. K., Li, M., and Sulakvelidze, A. (2015). Bacteriophage Administration Significantly Reduces Shigella Colonization and Shedding by Shigella-challenged Mice Without Deleterious Side Effects and Distortions in the Gut Microbiota. Bacteriophage 5, e1088124. doi: 10.1080/ 21597081.2015.1088124

Majewska, J., Beta, W., Lecion, D., Hodyra-Stefaniak, K., Klopot, A., Kazmierczak, Z., et al. (2015). Oral Application of T4 Phage Induces Weak Antibody Production in the Gut and in the Blood. Viruses 7, 4783-4799. doi: 10.3390/v7082845

Manrique, P., Bolduc, B., Walk, S. T., van der Oost, J., de Vos, W. M., and Young, M. J. (2016). Healthy Human Gut Phageome. Proc. Natl. Acad. Sci. U. S. A. 113, 10400-10405. doi: 10.1073/pnas.1601060113

Ma, C., Pan, N., Chen, Z., Liu, Z., Gong, G., and Ma, A. (2014). Geographical Diversity of Streptococcus Thermophilus Phages in Chinese Yoghurt Plants. Int. Dairy J. 35, 32-37. doi: 10.1016/j.idairyj.2013.10.007

Marchetti, G., Bellistrì, G. M., Borghi, E., Tincati, C., Ferramosca, S., La Francesca, M., et al. (2008). Microbial Translocation Is Associated With Sustained Failure in CD4+ T-Cell Reconstitution in HIV-Infected Patients on Long-Term Highly Active Antiretroviral Therapy. AIDS 22 (15), 2035-2038. doi: 10.1097/ QAD.0b013e3283112d29

Marco, M. B., Moineau, S., and Quiberoni, A. (2012). Bacteriophages and Dairy Fermentations. Bacteriophage 2, 149-158. doi: 10.4161/bact.21868

Martinez-Hernandez, F., Fornas, O., Gomez, M. L., Bolduc, B., de La Cruz Peña, M. J., Martínez, J. M., et al. (2017). Single-Virus Genomics Reveals Hidden Cosmopolitan and Abundant Viruses. Nat. Commun. 8, 1-13. doi: 10.1038/ ncomms 15892

Martinez-Medina, M., Aldeguer, X., Gonzalez-Huix, F., Acero, D., and Garcia-Gil, L. J. (2006). Abnormal Microbiota Composition in the Ileocolonic Mucosa of Crohn's Disease Patients as Revealed by Polymerase Chain ReactionDenaturing Gradient Gel Electrophoresis. Inflammation Bowel Dis. 12, 1136-1145. doi: 10.1097/01.mib.0000235828.09305.0c

Martin, R., Langa, S., Reviriego, C., Jiminez, E., Marin, M. L., Xaus, J., et al. (2003). Human Milk Is a Source of Lactic Acid Bacteria for the Infant Gut. J. Pediatr. 143, 754-758. doi: 10.1016/j.jpeds.2003.09.028

Maslov, S., and Sneppen, K. (2017). Population Cycles and Species Diversity in Dynamic Kill-the-Winner Model of Microbial Ecosystems. Sci. Rep. 7:39642. doi: $10.1038 /$ srep 39642

Mattila, S., Ruotsalainen, P., and Jalasvuori, M. (2015). On-Demand Isolation of Bacteriophages Against Drug-Resistant Bacteria for Personalized Phage Therapy. Front. Microbiol. 6, 1271. doi: 10.3389/fmicb.2015.01271

Ma, Y., You, X., Mai, G., Tokuyasu, T., and Liu, C. (2018). A Human Gut Phage Catalog Correlates the Gut Phageome With Type 2 Diabetes. Microbiome 6, 24. doi: 10.1186/s40168-018-0410-y

McCann, A., Ryan, F. J., Stockdale, S. R., Dalmasso, M., Blake, T., Ryan, C. A., et al. (2018). Viromes of One Year Old Infants Reveal the Impact of Birth Mode on Microbiome Diversity. PeerJ 6, e4694. doi: 10.7717/peerj.4694

McIntyre, K., Heap, H. A., Davey, G. P., and Limsowtin, G. K. (1991). The Distribution of Lactococcal Bacteriophage in the Environment of a Cheese Manufacturing Plant. Int. Dairy J. 1, 183-197. doi: 10.1016/0958-6946(91)90010-6

McNair, K., Zhou, C., Dinsdale, E. A., Souza, B., and Edwards, R. A. (2019). PHANOTATE: A Novel Approach to Gene Identification in Phage Genomes. Bioinformatics 35, 4537-4542. doi: 10.1093/bioinformatics/btz265

Méndez, J., Audicana, A., Cancer, M., Isern, A., Llaneza, J., Moreno, B., et al. (2004). Assessment of Drinking Water Quality Using Indicator Bacteria and Bacteriophages. J. Water Health 2, 201-214. doi: 10.2166/wh.2004.0018
Michail, S., Durbin, M., Turner, D., Griffiths, A. M., Mack, D. R., Hyams, J., et al. (2012). Alterations in the Gut Microbiome of Children With Severe Ulcerative Colitis. Inflammation Bowel Dis. 18, 1799-1808. doi: 10.1002/ibd.22860

Miernikiewicz, P., Klopot, A., Soluch, R., Szkuta, P., Keska, W., Hodyra-Stefaniak, K., et al. (2016). T4 Phage Tail Adhesin Gp12 Counteracts LPS-Induced Inflammation In Vivo. Front. Microbiol. 7, 1112. doi: 10.3389/fmicb.2016.01112

Mills, S., Shanahan, F., Stanton, C., Hill, C., Coffey, A., and Ross, R. P. (2013). Movers and Shakers: Influence of Bacteriophages in Shaping the Mammalian Gut Microbiota. Gut Microbes 4, 4-16. doi: 10.4161/gmic.22371

Minot, S., Bryson, A., Chehoud, C., Wu, G. D., Lewis, J. D., and Bushman, F. D. (2013). Rapid Evolution of the Human Gut Virome. Proc. Natl. Acad. Sci. U. S. A. 110, 12450-12455. doi: 10.1073/pnas. 1300833110

Minot, S., Grunberg, S., Wu, G. D., Lewis, J. D., and Bushman, F. D. (2012). Hypervariable Loci in the Human Gut Virome. Proc. Natl. Acad. Sci. U. S. A. 109, 3962-3966. doi: 10.1073/pnas.1119061109

Minot, S., Sinha, R., Chen, J., Li, H., Keilbaugh, S. A., Wu, G. D., et al. (2011). The Human Gut Virome: Inter-Individual Variation and Dynamic Response to Diet. Genome Res. 21, 1616-1625. doi: 10.1101/gr.122705.111

Mirzaei, M. K., Khan, M. A. A., Ghosh, P., Taranu, Z. E., Taguer, M., Ru, J., et al. (2020). Bacteriophages Isolated From Stunted Children Can Regulate Gut Bacterial Communities in an Age-Specific Manner. Cell Host Microbe 27, 199212.e195. doi: 10.1016/j.chom.2020.01.004

Mirzaei, M. K., and Maurice, C. F. (2017). Ménage À Trois in the Human Gut: Interactions Between Host, Bacteria and Phages. Nat. Rev. Microbiol. 15, 397408. doi: 10.1038/nrmicro.2017.30

Modi, S. R., Lee, H. H., Spina, C. S., and Collins, J. J. (2013). Antibiotic Treatment Expands the Resistance Reservoir and Ecological Network of the Phage Metagenome. Nature 499, 219-222. doi: 10.1038/nature12212

Mokili, J. L., Rohwer, F., and Dutilh, B. E. (2012). Metagenomics and Future Perspectives in Virus Discovery. Curr. Opin. Virol. 2, 63-77. doi: 10.1016/ j.coviro.2011.12.004

Molineux, I. (1991). Host-Parasite Interactions: Recent Developments in the Genetics of Abortive Phage Infections. New Biol. 3, 230-236.

Monaco, C. L., Gootenberg, D. B., Zhao, G., Handley, S. A., Ghebremichael, M. S., Lim, E. S., et al. (2016). Altered Virome and Bacterial Microbiome in Human Immunodeficiency Virus-Associated Acquired Immunodeficiency Syndrome. Cell Host Microbe 19, 311-322. doi: 10.1016/j.chom.2016.02.011

Moye, Z. D., Woolston, J., and Sulakvelidze, A. (2018). Bacteriophage Applications for Food Production and Processing. Viruses 10, 205. doi: 10.3390/v10040205

Muniesa, M., Colomer-Lluch, M., and Jofre, J. (2013). Could Bacteriophages Transfer Antibiotic Resistance Genes From Environmental Bacteria to Human-Body Associated Bacterial Populations? Mobile Genet. Elements 3, 739-751. doi: 10.4161/mge.25847

Murphy, J., Mahony, J., Bonestroo, M., Nauta, A., and van Sinderen, D. (2014). Impact of Thermal and Biocidal Treatments on Lactococcal 936-Type Phages. Int. Dairy J. 34, 56-61. doi: 10.1016/j.idairyj.2013.06.011

Murphy, S. J., Ullman, T. A., and Abreu, M. T. (2008). Gut Microbes in Crohn's Disease: Getting to Know You Better? Am. J. Gastroenterol. 103, 397-398. doi: 10.1111/j.1572-0241.2007.01571.x

Nagpal, R., Mainali, R., Ahmadi, S., Wang, S., Singh, R., Kavanagh, K., et al. (2018). Gut Microbiome and Aging: Physiological and Mechanistic Insights. Nutr. Healthy Aging 4, 267-285. doi: 10.3233/NHA-170030

Nale, J. Y., Redgwell, T. A., Millard, A., and Clokie, M. R. J. (2018). Efficacy of an Optimised Bacteriophage Cocktail to Clear Clostridium Difficile in a Batch Fermentation Model. Antibiotics 7, 13. doi: 10.3390/antibiotics7010013

Nash, A. K., Auchtung, T. A., Wong, M. C., Smith, D. P., Gesell, J. R., Ross, M. C., et al. (2017). The Gut Mycobiome of the Human Microbiome Project Healthy Cohort. Microbiome 5, 153. doi: 10.1186/s40168-017-0373-4

Nayfach, S., Camargo, A. P., Schulz, F., Eloe-Fadrosh, E., Roux, S., and Kyrpides, N. C. (2020). Checkv Assesses the Quality and Completeness of MetagenomeAssembled Viral Genomes. Nat. Biotechnol. 39 (5), 578-585. doi: 10.1038/ s41587-020-00774-7

Nazik, H., Joubert, L.-M., Secor, P. R., Sweere, J. M., Bollyky, P. L., Sass, G., et al. (2017). Pseudomonas Phage Inhibition of Candida Albicans. Microbiology 163, 1568-1577. doi: 10.1099/mic.0.000539

Nguyen, S., Baker, K., Padman, B. S., Patwa, R., Dunstan, R. A., Weston, T. A., et al. (2017). Bacteriophage Transcytosis Provides a Mechanism to Cross Epithelial Cell Layers. mBio 8, e01874-e01817. doi: 10.1128/mBio.01874-17 
Nguyen, T. L., Vieira-Silva, S., Liston, A., and Raes, J. (2015). How Informative Is the Mouse for Human Gut Microbiota Research? Dis. Model Mech. 8, 1-16. doi: $10.1242 / \mathrm{dmm} .017400$

Nicol, C. G., Denby, L., Lopez-Franco, O., Masson, R., Halliday, C. A., Nicklin, S. A., et al. (2009). Use of In Vivo Phage Display to Engineer Novel Adenoviruses for Targeted Delivery to the Cardiac Vasculature. FEBS Lett. 583, 2100-2107. doi: 10.1016/j.febslet.2009.05.037

Nordström, K., and Forsgren, A. (1974). Effect of Protein A on Adsorption of Bacteriophages to Staphylococcus Aureus. J. Virol. 14, 198-202. doi: 10.1128/ JVI.14.2.198-202.1974

Norman, J. M., Handley, S. A., Baldridge, M. T., Droit, L., Liu, C. Y., Keller, B. C., et al. (2015). Disease-Specific Alterations in the Enteric Virome in Inflammatory Bowel Disease. Cell 160, 447-460. doi: 10.1016/j.cell.2015.01.002

Norman, J. M., Handley, S. A., and Virgin, H. W. (2014). Kingdom-Agnostic Metagenomics and the Importance of Complete Characterization of Enteric Microbial Communities. Gastroenterology 146, 1459-1469. doi: 10.1053/ j.gastro.2014.02.001

Ofir, G., Melamed, S., Sberro, H., Mukamel, Z., Silverman, S., Yaakov, G., et al. (2018). DISARM Is a Widespread Bacterial Defence System With Broad AntiPhage Activities. Nat. Microbiol. 3, 90-98. doi: 10.1038/s41564-017-0051-0

Ogilvie, L. A., Bowler, L. D., Caplin, J., Dedi, C., Diston, D., Cheek, E., et al. (2013). Genome Signature-Based Dissection of Human Gut Metagenomes to Extract Subliminal Viral Sequences. Nat. Commun. 4, 2420. doi: 10.1038/ncomms3420

Ott, S. J., Waetzig, G. H., Rehman, A., Moltzau-Anderson, J., Bharti, R., Grasis, J. A., et al. (2017). Efficacy of Sterile Fecal Filtrate Transfer for Treating Patients With Clostridium Difficile Infection. Gastroenterology 152, 799-811.e797. doi: 10.1053/j.gastro.2016.11.010

Pannaraj, P. S., Ly, M., Cerini, C., Saavedra, M., Aldrovandi, G. M., Saboory, A. A., et al. (2018). Shared and Distinct Features of Human Milk and Infant Stool Viromes. Front. Microbiol. 9, 1162. doi: 10.3389/fmicb.2018.01162

Park, K., Cha, K. E., and Myung, H. (2014). Observation of Inflammatory Responses in Mice Orally Fed With Bacteriophage T7. J. Appl. Microbiol. 117, 627-633. doi: 10.1111/jam.12565

Park, J. P., Cropek, D. M., and Banta, S. (2010). High Affinity Peptides for the Recognition of the Heart Disease Biomarker Troponin I Identified Using Phage Display. Biotechnol. Bioeng. 105, 678-686. doi: 10.1002/bit.22597

Park, H., Laffin, M. R., Jovel, J., Millan, B., Hyun, J. E., Hotte, N., et al. (2019). The Success of Fecal Microbial Transplantation in Clostridium Difficile Infection Correlates With Bacteriophage Relative Abundance in the Donor: A Retrospective Cohort Study. Gut Microbes 10, 676-687. doi: 10.1080/ 19490976.2019.1586037

Parracho, H. M., Bingham, M. O., Gibson, G. R., and McCartney, A. L. (2005). Differences Between the Gut Microflora of Children With Autistic Spectrum Disorders and That of Healthy Children. J. Med. Microbiol. 54, 987-991. doi: 10.1099/jmm.0.46101-0

Peankuch, E., and Kausche, G. A. (1940). Isolierung Und, Bermikroskopische Abbildung Eines Bakteriophagen. Die Naturwissenschaften. 28, 46-46. doi: 10.1007/bf01486932

Pecota, D. C., and Wood, T. K. (1996). Exclusion of T4 Phage by the Hok/Sok Killer Locus From Plasmid R1. J. Bacteriol. 178, 2044-2050. doi: 10.1128/ jb.178.7.2044-2050.1996

Pedruzzi, I., Rosenbusch, J. P., and Locher, K. P. (1998). Inactivation In Vitro of the Escherichia Coli Outer Membrane Protein FhuA by a Phage T5-encoded Lipoprotein. FEMS Microbiol. Lett. 168, 119-125. doi: 10.1111/j.15746968.1998.tb13264.x

Pelzek, A. J., Schuch, R., Schmitz, J. E., and Fischetti, V. A. (2013). Isolation of Bacteriophages From Environmental Sources, and Creation and Functional Screening of Phage Dna Libraries. Curr. Protoc. Essent. Lab. Tech. 7, 13.13.1113.13.35. doi: 10.1002/9780470089941.et1303s07

Perez-Brocal, V., Garcia-Lopez, R., Nos, P., Beltran, B., Moret, I., and Moya, A. (2015). Metagenomic Analysis of Crohn's Disease Patients Identifies Changes in the Virome and Microbiome Related to Disease Status and Therapy, and Detects Potential Interactions and Biomarkers. Inflammation Bowel Dis. 21, 2515-2532. doi: 10.1097/MIB.0000000000000549

Pires, D. P., Oliveira, H., Melo, L. D. R., Sillankorva, S., and Azeredo, J. (2016). Bacteriophage-Encoded Depolymerases: Their Diversity and Biotechnological Applications. Appl. Microbiol. Biotechnol. 100, 2141-2151. doi: 10.1007/ s00253-015-7247-0
Potapov, E. V., Trampuz, A., and Mulzer, J. (2020). Treatment of Chronic Left Ventricular Assist Device Infection With Local Application of Bacteriophages. Eur. J. Cardiothorac Surg. 57, 1003-1004. doi: 10.1093/ejcts/ezz295

Powell, S., Szklarczyk, D., Trachana, K., Roth, A., Kuhn, M., Muller, J., et al. (2012). eggNOG v3. 0: Orthologous Groups Covering 1133 Organisms at 41 Different Taxonomic Ranges. Nucleic Acids Res. 40, D284-D289. doi: 10.1093/nar/ gkr1060

Qin, J., Li, Y., Cai, Z., Li, S., Zhu, J., Zhang, F., et al. (2012). A Metagenome-Wide Association Study of Gut Microbiota in Type 2 Diabetes. Nature 490, 55-60. doi: $10.1038 /$ nature 11450

Qin, J., Li, R., Raes, J., Arumugam, M., Burgdorf, K. S., Manichanh, C., et al. (2010). A Human Gut Microbial Gene Catalogue Established by Metagenomic Sequencing. Nature 464, 59-65. doi: 10.1038/nature08821

Quiberoni, A., Tremblay, D., Ackermann, H. W., Moineau, S., and Reinheimer, J. A. (2006). Diversity of Streptococcus Thermophilus Phages in a LargeProduction Cheese Factory in Argentina. J. Dairy Sci. 89, 3791-3799. doi: 10.3168/jds.S0022-0302(06)72420-1

Quiros, P., Colomer-Lluch, M., Martinez-Castillo, A., Miro, E., Argente, M., Jofre, J., et al. (2014). Antibiotic Resistance Genes in the Bacteriophage DNA Fraction of Human Fecal Samples. Antimicrob. Agents Chemother. 58, 606609. doi: 10.1128/AAC.01684-13

Røder, H. L., Sørensen, S. J., and Burmølle, M. (2016). Studying Bacterial Multispecies Biofilms: Where to Start? Trends Microbiol. 24, 503-513. doi: 10.1016/j.tim.2016.02.019

Rasmussen, T. S., Mentzel, C. M. J., Kot, W., Castro-Mejia, J. L., Zuffa, S., Swann, J. R., et al. (2020). Faecal Virome Transplantation Decreases Symptoms of Type 2 Diabetes and Obesity in a Murine Model. Gut 69, 2122-2130. doi: 10.1136/ gutjnl-2019-320005

Ren, J., Song, K., Deng, C., Ahlgren, N. A., Fuhrman, J. A., Li, Y., et al. (2020). Identifying Viruses From Metagenomic Data Using Deep Learning. Quant Biol 8, 64-77. doi: 10.1007/s40484-019-0187-4

Ren, J., Ahlgren, N. A., Lu, Y. Y., Fuhrman, J. A., and Sun, F. (2017). VirFinder: A Novel K-Mer Based Tool for Identifying Viral Sequences From Assembled Metagenomic Data. Microbiome 5, 1-20. doi: 10.1186/s40168-017-0283-5

Reyes, A., Blanton, L. V., Cao, S., Zhao, G., Manary, M., Trehan, I., et al. (2015). Gut DNA Viromes of Malawian Twins Discordant for Severe Acute Malnutrition. Proc. Natl. Acad. Sci. U. S. A. 112, 11941-11946. doi: 10.1073/ pnas. 1514285112

Reyes, A., Haynes, M., Hanson, N., Angly, F. E., Heath, A. C., Rohwer, F., et al. (2010). Viruses in the Faecal Microbiota of Monozygotic Twins and Their Mothers. Nature 466, 334-338. doi: 10.1038/nature09199

Reyes, A., Semenkovich, N. P., Whiteson, K., Rohwer, F., and Gordon, J. I. (2012). Going Viral: Next-Generation Sequencing Applied to Phage Populations in the Human Gut. Nat. Rev. Microbiol. 10, 607-617. doi: 10.1038/nrmicro2853

Reyes, A., Wu, M., McNulty, N. P., Rohwer, F. L., and Gordon, J. I. (2013). Gnotobiotic Mouse Model of Phage-Bacterial Host Dynamics in the Human Gut. Proc. Natl. Acad. Sci. U. S. A. 110, 20236-20241. doi: 10.1073/ pnas. 1319470110

Rice, S. A., Tan, C. H., Mikkelsen, P. J., Kung, V., Woo, J., Tay, M., et al. (2009). The Biofilm Life Cycle and Virulence of Pseudomonas Aeruginosa Are Dependent on a Filamentous Prophage. ISME J. 3, 271-282. doi: 10.1038/ ismej.2008.109

Rossmann, F. S., Racek, T., Wobser, D., Puchalka, J., Rabener, E. M., Reiger, M., et al. (2015). Phage-Mediated Dispersal of Biofilm and Distribution of Bacterial Virulence Genes Is Induced by Quorum Sensing. PloS Pathogens 11 (2), e1004653. doi: 10.1371/journal.ppat.1004653

Roux, S., Emerson, J. B., Eloe-Fadrosh, E. A., and Sullivan, M. B. (2017). Benchmarking Viromics: An in Silico Evaluation of Metagenome-Enabled Estimates of Viral Community Composition and Diversity. PeerJ 5, e3817. doi: $10.7717 /$ peerj. 3817

Roux, S., Páez-Espino, D., Chen, I.-M. A., Palaniappan, K., Ratner, A., Chu, K., et al. (2021). Img/Vr v3: An Integrated Ecological and Evolutionary Framework for Interrogating Genomes of Uncultivated Viruses. Nucleic Acids Res. 49, D764-D775. doi: 10.1093/nar/gkaa946

Ruska, H. (1940). Die Sichtbarmachung Der Bakteriophagen Lyse Im Bermikroskop. Die Naturwissenschaften. 28, 45-46. doi: 10.1007/bf01486931

Sørensen, M. C. H., van Alphen, L. B., Harboe, A., Li, J., Christensen, B. B., Szymanski, C. M., et al. (2011). Bacteriophage F336 Recognizes the Capsular 
Phosphoramidate Modification of Campylobacter Jejuni NCTC11168. J. Bacteriol. 193, 6742-6749. doi: 10.1128/JB.05276-11

Sandek, A., Bauditz, J., Swidsinski, A., Buhner, S., Weber-Eibel, J., von Haehling, S., et al. (2007). Altered Intestinal Function in Patients With Chronic Heart Failure. J. Am. Coll. Cardiol. 50, 1561-1569. doi: 10.1016/j.jacc.2007.07.016

Sarker, S. A., Sultana, S., Reuteler, G., Moine, D., Descombes, P., Charton, F., et al. (2016). Oral Phage Therapy of Acute Bacterial Diarrhea With Two Coliphage Preparations: A Randomized Trial in Children From Bangladesh. EBioMedicine 4, 124-137. doi: 10.1016/j.ebiom.2015.12.023

Schuch, R., and Fischetti, V. A. (2009). The Secret Life of the Anthrax Agent Bacillus Anthracis: Bacteriophage-Mediated Ecological Adaptations. PloS One 4, e6532. doi: 10.1371/journal.pone.0006532

Schulfer, A., Santiago-Rodriguez, T. M., Ly, M., Borin, J. M., Chopyk, J., Blaser, M. J., et al. (2020). Fecal Viral Community Responses to High-Fat Diet in Mice. mSphere 5, e00833-e00819. doi: 10.1128/mSphere.00833-19

Seemann, T. (2014). Prokka: Rapid Prokaryotic Genome Annotation. Bioinformatics 30, 2068-2069. doi: 10.1093/bioinformatics/btu153

Sender, R., Fuchs, S., and Milo, R. (2016). Revised Estimates for the Number of Human and Bacteria Cells in the Body. PloS Biol. 14, e1002533. doi: 10.1371/ journal.pbio. 1002533

Serwer, P., Hayes, S. J., Thomas, J. A., Demeler, B., and Hardies, S. C. (2009). "Isolation of Novel Large and Aggregating Bacteriophages". In: Bacteriophages (Berlin, Germany: Springer), 55-66. doi: 10.1007/978-1-60327-164-6_6

Shaffer, M., Borton, M. A., McGivern, B. B., Zayed, A. A., La Rosa, S. L., Solden, L. M., et al. (2020). DRAM for Distilling Microbial Metabolism to Automate the Curation of Microbiome Function. Nucleic Acids Res. 48, 8883-8900. doi: 10.1093/nar/gkaa621

Sharon, I., Morowitz, M. J., Thomas, B. C., Costello, E. K., Relman, D. A., and Banfield, J. F. (2013). Time Series Community Genomics Analysis Reveals Rapid Shifts in Bacterial Species, Strains, and Phage During Infant Gut Colonization. Genome Res. 23, 111-120. doi: 10.1101/gr.142315.112

Shen, M., Yang, Y., Shen, W., Cen, L., McLean, J. S., Shi, W., et al. (2018). A Linear Plasmid-Like Prophage of Actinomyces Odontolyticus Promotes Biofilm Assembly. Appl. Environ. Microbiol. 84, e01263-e01218. doi: 10.1128/ AEM.01263-18

Sherwin, E., Sandhu, K. V., Dinan, T. G., and Cryan, J. F. (2016). May the Force be With You: The Light and Dark Sides of the Microbiota-Gut-Brain Axis in Neuropsychiatry. CNS Drugs 30, 1019-1041. doi: 10.1007/s40263-016-0370-3

Shimamura, T., Jeng, D., Lucas, A., and Essani, K. (2012). Suppression of Neointimal Hyperplasia Following Angioplasty-Induced Vascular Injury in Pigs Infected With Swinepox Virus. Open Virol. J. 6, 91-96. doi: 10.2174/ 1874357901206010091

Shkoporov, A. N., Clooney, A. G., Sutton, T. D., Ryan, F. J., Daly, K. M., Nolan, J. A., et al. (2019). The Human Gut Virome Is Highly Diverse, Stable, and Individual Specific. Cell Host Microbe 26, 527-541. e525. doi: 10.1016/ j.chom.2019.09.009.

Shkoporov, A. N., and Hill, C. (2019). Bacteriophages of the Human Gut: The "Known Unknown" of the Microbiome. Cell Host Microbe 25, 195-209. doi: 10.1016/j.chom.2019.01.017

Shkoporov, A. N., Khokhlova, E. V., Fitzgerald, C. B., Stockdale, S. R., Draper, L. A., Ross, R. P., et al. (2018a). PhiCrAss001 Represents the Most Abundant Bacteriophage Family in the Human Gut and Infects Bacteroides Intestinalis. Nat. Commun. 9, 4781. doi: 10.1038/s41467-018-07225-7

Shkoporov, A. N., Ryan, F. J., Draper, L. A., Forde, A., Stockdale, S. R., Daly, K. M., et al. (2018b). Reproducible Protocols for Metagenomic Analysis of Human Faecal Phageomes. Microbiome 6, 68. doi: 10.1186/s40168-018-0446-z

Sieradzki, E. T., Ignacio-Espinoza, J. C., Needham, D. M., Fichot, E. B., and Fuhrman, J. A. (2019). Dynamic Marine Viral Infections and Major Contribution to Photosynthetic Processes Shown by Spatiotemporal Picoplankton Metatranscriptomes. Nat. Commun. 10, 1169. doi: 10.1038/ s41467-019-09106-z

Sillankorva, S. M., Oliveira, H., and Azeredo, J. (2012). Bacteriophages and Their Role in Food Safety. Int. J. Microbiol. 2012, 13. doi: 10.1155/2012/ 863945

Silveira, C. B., Coutinho, F. H., Cavalcanti, G. S., Benler, S., Doane, M. P., Dinsdale, E. A., et al. (2020). Genomic and Ecological Attributes of Marine Bacteriophages Encoding Bacterial Virulence Genes. BMC Genom. 21, 1-11. doi: 10.1186/s12864-020-6523-2
Silveira, C. B., and Rohwer, F. L. (2016). Piggyback-the-Winner in HostAssociated Microbial Communities. NPJ Biofilms Microbiomes 2, 16010. doi: 10.1038/npjbiofilms.2016.10

Singh, R. K., Chang, H. W., Yan, D., Lee, K. M., Ucmak, D., Wong, K., et al. (2017). Influence of Diet on the Gut Microbiome and Implications for Human Health. J. Transl. Med. 15, 73. doi: 10.1186/s12967-017-1175-y

Siranosian, B. A., Tamburini, F. B., Sherlock, G., and Bhatt, A. S. (2020). Acquisition, Transmission and Strain Diversity of Human Gut-Colonizing crAss-like Phages. Nat. Commun. 11, 280. doi: 10.1038/s41467-019-14103-3

Sjöström, J., Löfdahl, S., and Philipson, L. (1978). Biological Characteristics of a Type I Restriction-Modification System in Staphylococcus Aureus. J. Bacteriol. 133, 1144-1149. doi: 10.1128/JB.133.3.1144-1149.1978

Skewes-Cox, P., Sharpton, T. J., Pollard, K. S., and DeRisi, J. L. (2014). Profile Hidden Markov Models for the Detection of Viruses Within Metagenomic Sequence Data. PloS One 9, e105067. doi: 10.1371/journal.pone.0105067

Starr, E. P., Nuccio, E. E., Pett-Ridge, J., Banfield, J. F., and Firestone, M. K. (2019). Metatranscriptomic Reconstruction Reveals RNA Viruses With the Potential to Shape Carbon Cycling in Soil. Proc. Natl. Acad. Sci. U. S. A. 116, 2590025908. doi: 10.1073/pnas.1908291116

Stern, A., Mick, E., Tirosh, I., Sagy, O., and Sorek, R. (2012). CRISPR Targeting Reveals a Reservoir of Common Phages Associated With the Human Gut Microbiome. Genome Res. 22, 1985-1994. doi: 10.1101/gr.138297.112

Stummeyer, K., Schwarzer, D., Claus, H., Vogel, U., Gerardy-Schahn, R., and Mühlenhoff, M. (2006). Evolution of Bacteriophages Infecting Encapsulated Bacteria: Lessons From Escherichia Coli K1-Specific Phages. Mol. Microbiol. 60, 1123-1135. doi: 10.1111/j.1365-2958.2006.05173.x

Suarez, V. B., Quiberoni, A., Binetti, A. G., and Reinheimer, J. A. (2002). Thermophilic Lactic Acid Bacteria Phages Isolated From Argentinian Dairy Industries. J. Food Prot. 65, 1597-1604. doi: 10.4315/0362-028x-65.10.1597

Sun, X., Göhler, A., Heller, K. J., and Neve, H. (2006). The ltp Gene of Temperate Streptococcus thermophilus Phage TP-J34 Confers Superinfection Exclusion to Streptococcus thermophilus and Lactococcus lactis. Virology 350(1), 146-157. doi: $10.1016 /$ j.virol.2006.03.001

Sutton, T. D. S., and Hill, C. (2019). Gut Bacteriophage: Current Understanding and Challenges. Front. Endocrinol. 10, 784. doi: 10.3389/fendo.2019.00784

Swidsinski, A., Weber, J., Loening-Baucke, V., Hale, L. P., and Lochs, H. (2005). Spatial Organization and Composition of the Mucosal Flora in Patients With Inflammatory Bowel Disease. J. Clin. Microbiol. 43, 3380-3389. doi: 10.1128/ JCM.43.7.3380-3389.2005

Tanaka, M., and Nakayama, J. (2017). Development of the Gut Microbiota in Infancy and Its Impact on Health in Later Life. Allergol. Int. 66, 515-522. doi: 10.1016/j.alit.2017.07.010

Tetz, G., Brown, S. M., Hao, Y., and Tetz, V. (2019). Type 1 Diabetes: An Association Between Autoimmunity, the Dynamics of Gut Amyloid-Producing E. Coli and Their Phages. Sci. Rep. 9, 9685. doi: 10.1038/s41598-019-46087-x

Tetz, G. V., Ruggles, K. V., Zhou, H., Heguy, A., Tsirigos, A., and Tetz, V. (2017). Bacteriophages as Potential New Mammalian Pathogens. Sci. Rep. 7, 7043. doi: 10.1038/s41598-017-07278-6

Tetz, G., and Tetz, V. (2016). Bacteriophage Infections of Microbiota Can Lead to Leaky Gut in an Experimental Rodent Model. Gut Pathog. 8, 33. doi: 10.1186/ s13099-016-0109-1

Thurber, R. V., Haynes, M., Breitbart, M., Wegley, L., and Rohwer, F. (2009). Laboratory Procedures to Generate Viral Metagenomes. Nat. Protoc. 4, 470483. doi: $10.1038 /$ nprot.2009.10

Trevors, K., Holley, R., and Kempton, A. (1984). Effect of Bacteriophage on the Activity of Lactic Acid Starter Cultures Used in the Production of Fermented Sausage. J. Food Sci. 49, 650-651. doi: 10.1111/j.1365-2621.1984.tb12492.x

Trifonov, V., and Rabadan, R. (2010). Frequency Analysis Techniques for Identification of Viral Genetic Data. MBio 1 (3), e00156-10. doi: 10.1128/ mBio.00156-10

Twort, F. W. (1961). An Investigation on the Nature of Ultra-Microscopic Viruses. Acta Kravsi 186 (4814), 1241-1243. doi: 10.1016/S0140-6736(01)20383-3

Tytgat, H. L. P., Nobrega, F. L., van der Oost, J., and de Vos, W. M. (2019). Bowel Biofilms: Tipping Points Between a Healthy and Compromised Gut? Trends Microbiol. 27, 17-25. doi: 10.1016/j.tim.2018.08.009

Uhl, M., and Miller, J. (1996). Integration of Multiple Domains in a TwoComponent Sensor Protein: The Bordetella Pertussis BvgAS Phosphorelay. EMBO J. 15, 1028-1036. doi: 10.1002/j.1460-2075.1996.tb00440.x 
Van Valen, L. (1973) A New Evolutionary Law. Available at: https://www.mn.uio. no/cees/english/services/van-valen/evolutionary-theory/volume-1/vol-1-no-1pages-1-30-l-van-valen-a-new-evolutionary-law.pdf.

Vázquez-Castellanos, J. F., García-López, R., Pérez-Brocal, V., Pignatelli, M., and Moya, A. (2014). Comparison of Different Assembly and Annotation Tools on Analysis of Simulated Viral Metagenomic Communities in the Gut. BMC Genom. 15, 1-20. doi: 10.1186/1471-2164-15-37

Ventura, M., Canchaya, C., Pridmore, D., Berger, B., and Brüssow, H. (2003). Integration and Distribution of Lactobacillus Johnsonii Prophages. J. Bacteriol. 185, 4603-4608. doi: 10.1128/JB.185.15.4603-4608.2003

Vieira-Silva, S., Falony, G., Darzi, Y., Lima-Mendez, G., Garcia Yunta, R., Okuda, S., et al. (2016). Species-function Relationships Shape Ecological Properties of the Human Gut Microbiome. Nat. Microbiol. 1, 16088. doi: 10.1038/ nmicrobiol.2016.88

Wagner, J., Maksimovic, J., Farries, G., Sim, W. H., Bishop, R. F., Cameron, D. J., et al. (2013). Bacteriophages in Gut Samples From Pediatric Crohn's Disease Patients: Metagenomic Analysis Using 454 Pyrosequencing. Inflammation Bowel Dis. 19, 1598-1608. doi: 10.1097/MIB.0b013e318292477c

Waller, A. S., Yamada, T., Kristensen, D. M., Kultima, J. R., Sunagawa, S., Koonin, E. V., et al. (2014). Classification and Quantification of Bacteriophage Taxa in Human Gut Metagenomes. ISME J. 8, 1391-1402. doi: 10.1038/ismej.2014.30

Wang, X., Kim, Y., Ma, Q., Hong, S. H., Pokusaeva, K., Sturino, J. M., et al. (2010). Cryptic Prophages Help Bacteria Cope With Adverse Environments. Nat. Commun. 1, 147. doi: 10.1038/ncomms1146

Wang, Z., Klipfell, E., Bennett, B. J., Koeth, R., Levison, B. S., Dugar, B., et al. (2011). Gut Flora Metabolism of Phosphatidylcholine Promotes Cardiovascular Disease. Nature 472, 57-63. doi: 10.1038/nature09922

Warren, R. A. (1980). Modified Bases in Bacteriophage DNAs. Annu. Rev. Microbiol. 34, 137-158. doi: 10.1146/annurev.mi.34.100180.001033

Weitz, J. S., and Dushoff, J. (2007). Alternative Stable States in Host-Phage Dynamics. Theor. Ecol. 1, 13-19. doi: 10.1007/s12080-007-0001-1

Wilhelm, S. W., and Suttle, C. A. (1999). Viruses and Nutrient Cycles in the Sea: Viruses Play Critical Roles in the Structure and Function of Aquatic Food Webs. Bioscience 49, 781-788. doi: 10.2307/1313569

Williams, J. W., Huang, L. H., and Randolph, G. J. (2019). Cytokine Circuits in Cardiovascular Disease. Immunity 50, 941-954. doi: 10.1016/j.immuni. 2019.03.007

Williamson, S. J., Rusch, D. B., Yooseph, S., Halpern, A. L., Heidelberg, K. B., Glass, J. I., et al. (2008). The Sorcerer II Global Ocean Sampling Expedition: Metagenomic Characterization of Viruses Within Aquatic Microbial Samples. PloS One 3, e1456. doi: 10.1371/journal.pone.0001456

Wommack, K. E., and Colwell, R. R. (2000). Virioplankton: Viruses in Aquatic Ecosystems. Microbiol. Mol. Biol. Rev. 64, 69-114. doi: 10.1128/mmbr.64.1.69114.2000

Yarur, A. J., Deshpande, A. R., Pechman, D. M., Tamariz, L., Abreu, M. T., and Sussman, D. A. (2011). Inflammatory Bowel Disease Is Associated With an Increased Incidence of Cardiovascular Events. Am. J. Gastroenterol. 106, 741747. doi: 10.1038/ajg.2011.63

Yassour, M., Vatanen, T., Siljander, H., Hämäläinen, A.-M., Härkönen, T., Ryhänen, S. J., et al. (2016). Natural History of the Infant Gut Microbiome and Impact of Antibiotic Treatment on Bacterial Strain Diversity and Stability. Sci. Transl. Med. 8, 343ra381-343ra381. doi: 10.1126/scitranslmed.aad0917

Yoon, S. S., Barrangou-Poueys, R., Breidt, F. Jr., Klaenhammer, T. R., and Fleming, H. P. (2002). Isolation and Characterization of Bacteriophages From
Fermenting Sauerkraut. Appl. Environ. Microbiol. 68, 973-976. doi: 10.1128/ aem.68.2.973-976.2002

Yutin, N., Makarova, K. S., Gussow, A. B., Krupovic, M., Segall, A., Edwards, R. A., et al. (2018). Discovery of an Expansive Bacteriophage Family That Includes the Most Abundant Viruses From the Human Gut. Nat. Microbiol. 3, 38-46. doi: 10.1038/s41564-017-0053-y

Zablocki, O., Jang, H. B., Bolduc, B., and Sullivan, M. B. (2019). "vConTACT 2: A Tool to Automate Genome-Based Prokaryotic Viral Taxonomy," in Proceedings of the Plant and Animal Genome XXVII Conference, January 1216, 2019 (San Diego, CA, United States of America: PAG).

Zhang, T., Breitbart, M., Lee, W. H., Run, J.-Q., Wei, C. L., Soh, S. W. L., et al. (2005). RNA Viral Community in Human Feces: Prevalence of Plant Pathogenic Viruses. PloS Biol. 4, e3. doi: 10.1371/journal.pbio.0040003

Zhang, X., Guo, H., Jin, L., Czornyj, E., Hodes, A., Hui, W. H., et al. (2013). A New Topology of the HK97-like Fold Revealed in Bordetella Bacteriophage by cryoEM at 3.5 A Resolution. Elife 2, e01299. doi: 10.7554/eLife.01299

Zhao, P. Y., Baek, H. Y., and Kim, I. H. (2012). Effects of Bacteriophage Supplementation on Egg Performance, Egg Quality, Excreta Microflora, and Moisture Content in Laying Hens. Asian-Australas J. Anim. Sci. 25, 1015-1020. doi: 10.5713 /ajas.2012.12026

Zhao, G., Droit, L., Gilbert, M. H., Schiro, F. R., Didier, P. J., Si, X., et al. (2019). Virome Biogeography in the Lower Gastrointestinal Tract of Rhesus Macaques With Chronic Diarrhea. Virology 527, 77-88. doi: 10.1016/j.virol. 2018.10.001

Zhao, G., Vatanen, T., Droit, L., Park, A., Kostic, A. D., Poon, T. W., Vlamakis, H., et al. (2017). Intestinal Virome Changes Precede Autoimmunity in Type I Diabetes-Susceptible Children. Proc. Nat. Acad. Sci. 114 (30), E6166-E6175. doi: $10.1073 /$ pnas.1706359114

Zilberman-Schapira, G., Zmora, N., Itav, S., Bashiardes, S., Elinav, H., and Elinav, E. (2016). The Gut Microbiome in Human Immunodeficiency Virus Infection. BMC Med. 14, 83. doi: 10.1186/s12916-016-0625-3

Zoetendal, E. G., Raes, J., Van Den Bogert, B., Arumugam, M., Booijink, C. C., Troost, F. J., et al. (2012). The Human Small Intestinal Microbiota Is Driven by Rapid Uptake and Conversion of Simple Carbohydrates. ISME J. 6, 1415-1426. doi: 10.1038/ismej.2011.212

Zuo, T., Lu, X. J., Zhang, Y., Cheung, C. P., Lam, S., Zhang, F., et al. (2019). Gut Mucosal Virome Alterations in Ulcerative Colitis. Gut 68, 1169-1179. doi: 10.1136/gutjnl-2018-318131

Zuo, T., Wong, S. H., Lam, K., Lui, R., Cheung, K., Tang, W., et al. (2018). Bacteriophage Transfer During Faecal Microbiota Transplantation in Clostridium Difficile Infection Is Associated With Treatment Outcome. Gut 67, 634-643. doi: 10.1136/gutjnl-2017-313952

Conflict of Interest: The authors declare that the research was conducted in the absence of any commercial or financial relationships that could be construed as a potential conflict of interest.

Copyright (c) 2021 Townsend, Kelly, Muscatt, Box, Hargraves, Lilley and Jameson. This is an open-access article distributed under the terms of the Creative Commons Attribution License (CC BY). The use, distribution or reproduction in other forums is permitted, provided the original author(s) and the copyright owner(s) are credited and that the original publication in this journal is cited, in accordance with accepted academic practice. No use, distribution or reproduction is permitted which does not comply with these terms. 